\title{
س
}

$>\mathrm{DE}$

$\stackrel{w 1}{\simeq}$ PÉDAGOGIE

\author{
Recherches en éducation
}

179 | avril-juin 2012

Varia

\section{Métacognition et croyances motivationnelles : un mariage de raison}

Metacognition and motivational beliefs: A marriage of convenience

Metacognición y creencias motivacionales: ¿Un matrimonio de conveniencia?

Metakognition und motivationale Überzeugungen: eine Vernunftehe

Jean-Louis Berger et Fredi Büchel

\section{(2) OpenEdition}

Journals

Édition électronique

URL : http://journals.openedition.org/rfp/3705

DOI : 10.4000/rfp.3705

ISSN : 2105-2913

Éditeur

ENS Éditions

Édition imprimée

Date de publication : 15 juin 2012

Pagination : $95-128$

ISBN : 978-2-84788-379-4

ISSN : 0556-7807

Référence électronique

Jean-Louis Berger et Fredi Büchel, « Métacognition et croyances motivationnelles : un mariage de raison », Revue française de pédagogie [En ligne], 179 | avril-juin 2012, mis en ligne le 15 juin 2015, consulté le 23 avril 2019. URL : http://journals.openedition.org/rfp/3705; DOI : 10.4000/rfp.3705 


\section{NOTE DE SYNTHÈSE}

\section{Métacognition et croyances motivationnelles : un mariage de raison Jean-Lovis Berger et Fredi Büchel}

Bien que l'importance des croyances motivationnelles pour l'apprentissage et la réussite scolaire soit passablement reconnue, les chercheurs ne se sont que récemment intéressés aux relations entre ces croyances et les aspects métacognitifs de l'apprentissage, notamment dans le cadre des modèles de l'apprentissage autorégulé. Nous proposons une synthèse des positions théoriques ainsi que d'un certain nombre d'études empiriques s'étant penchées sur les articulations entre métacognition et motivation. Pour conclure, les implications pour l'enseignement, l'apprentissage et les interventions de type éducation cognitive sont discutées.

Mots-clés (TESE) : développement cognitif, résolution de problème, confiance en soi, autonomie personnelle, compétences transversales générales, stratégie d'apprentissage.

\section{INTRODUCTION}

La motivation est reconnue comme essentielle dans le domaine de l'apprentissage en ce qu'elle constitue un déterminant important de la réussite ou de l'échec scolaire (Cosnefroy, 2011 ; Cosnefroy \& Fenouillet, 2009 ; Galand \& Bourgeois, 2006). Toutefois, les modèles du fonctionnement intellectuel dans le domaine de l'apprentissage sont souvent strictement composés d'aspects purement cognitifs et métacognitifs ${ }^{1}$ (voir par exemple Büchel, 1996 ; Nelson \& Narens, 1990 ; Schraw, 1998 ; Veenman, Elshout \& Meijer, 1997), car seuls ces derniers sont considérés comme prépondérants ${ }^{2}$. Dans cette note de synthèse, nous formulons, dans un premier temps, quelques critiques au sujet du traitement indépendant des domaines de la motivation et de la métacognition et nous relevons, pour ce faire, des arguments théoriques. Dans un deuxième temps, nous exposons des modèles récents de l'apprentissage autorégulé qui tentent d'articuler plusieurs aspects métacognitifs avec différentes croyances motivationnelles. Nous présentons, par la suite, une 
revue d'études empiriques en fonction des théories adoptées dans ce travail. Finalement, nous tirons de cette synthèse un certain nombre d'implications pour l'apprentissage et l'enseignement.

De récentes revues de littérature relatives au construit de la métacognition ont montré que trois composantes sont distinguables (Berger, 2008 ; Efklides, 2001, 2006, 2008 ; Pintrich, Wolters \& Baxter, 2000) : connaissances métacognitives, expériences métacognitives et stratégies métacognitives. Les connaissances métacognitives sont composées des connaissances de l'apprenant ${ }^{3}$ (enregistrées en mémoire à long terme) sur les stratégies cognitives, les caractéristiques des tâches, le fonctionnement de la cognition humaine et sur soi en tant qu'individu pensant (par exemple des connaissances comparatives de ses propres forces et faiblesses comme apprenant). Selon Paris, Lipson et Wixson (1983), ces connaissances peuvent être de type déclaratif (connaître une stratégie pour faciliter la résolution de problèmes mathématiques par exemple), procédural (savoir comment on peut appliquer cette stratégie) ou conditionnel (savoir dans quelles conditions et à quel moment on pourrait l'appliquer). Toutefois, différents auteurs (voir par exemple Büchel, 1991) ont argumenté qu'il n'est pas nécessaire de postuler des métaconnaissances conditionnelles. Une stratégie est par définition procédurale. Or une procédure inclut les conditions sous lesquelles elle peut être appliquée. VandenBos (2007), par exemple, définit une stratégie comme " a program of action designed to achieve a goal or accomplish a task ${ }^{4}$ » (p. 897). Dans cette définition, la spécificité du but ou de la tâche représente la condition d'application de la stratégie.

Les expériences métacognitives sont des sentiments et jugements subjectifs relatifs à l'entreprise cognitive " actuelle » de l'apprenant (qui est celle en cours lors de la réalisation de la tâche); elles constituent l'interface entre l'apprenant et la tâche. Ces expériences se produisent lorsque l'apprenant prend conscience de ses processus de pensée actuels. Leur fonction principale est de guider les processus de pensée d'un point de vue subjectif et du point de vue des connaissances spécifiques à la tâche, ceci afin d'influencer les stratégies métacognitives (Efklides, 2001). Elles prennent la forme de jugements tels que des jugements sur la difficulté de la tâche, des jugements de confiance sur l'exactitude de la solution ou la forme de sentiments tels que le sentiment de familiarité avec la tâche. Les stratégies métacognitives sont définies comme les décisions prises sur la base des expériences métacognitives, tant de façon consciente qu'inconsciente (Brown, 1987 ; Büchel \& Büchel, 2009 ; Perkins, Simmons \& Tishman, 1990). Deux types génériques d'activités sont considérés comme représentatifs des stratégies métacognitives : les activités d'anticipation et de planification réalisées avant la résolution d'une tâche (par exemple rechercher quelles pourraient être les difficultés potentielles, définir les étapes pour la résolution de la tâche, choisir des stratégies et prédire les résultats); les activités de vérification pendant et après la résolution d'une tâche (par exemple vérifier la compréhension, réviser les stratégies, voir Brown, 1987).

Les expériences métacognitives et les stratégies métacognitives sont fortement interdépendantes (Efklides, 2001 ; Nelson \& Narens, 1990). Selon le modèle de la métacognition développé par Nelson (1996 ; Nelson \& Narens, 1990), les processus cognitifs seraient divisés en au moins deux niveaux : le niveau dit « objet » se réfère aux actions et conduites du monde externe et le niveau « méta " consiste en une représentation du niveau objet, cette représentation spécifiant un but ainsi que la façon dont le niveau objet peut être utilisé afin de l'atteindre. Les deux niveaux du modèle s'informent réciproquement de leur fonctionnement par des flux d'informations nommés monitorage et contrôle. Le monitorage désigne l'information du niveau méta par le niveau objet quant à l'état de ce dernier, ce qui a pour fonction de mettre à jour l'état du modèle de niveau méta de la situation. À l'opposé, le contrôle 
désigne l'information, par le niveau méta, de ce qui doit être fait au niveau objet (par exemple, changer de stratégie ou allouer un certain temps d'étude). Le monitorage est inclus dans le concept d'expériences métacognitives alors que le contrôle fait partie des stratégies métacognitives.

Suivant la proposition de Pintrich, Marx et Boyle (1993), nous utiliserons dans ce texte les termes « croyances motivationnelles " pour désigner une série de construits tirés des théories sociocognitives de la motivation (voir Murphy \& Alexander, 2000, pour une synthèse de ces théories). Au même titre qu'ils disposent de conceptions sur la cognition, les apprenants développent également un certain nombre de connaissances et croyances au sujet de leur motivation (Boekaerts, 2010 ; Pintrich, 2002). Boekaerts (2010) définit les croyances motivationnelles comme des cognitions sur soi-même en relation à un sujet particulier, cognitions qui réfèrent à la connaissance et aux conceptions des apprenants du fonctionnement de leur propre système motivationnel dans les divers sujets. Leur rôle consiste à donner un sens aux tâches, aux situations d'apprentissage ainsi qu'au contexte d'apprentissage. Ainsi les croyances motivationnelles influencent-elles la propension à s'engager dans les tâches, ceci même si l'élève n'en est pas forcément conscient. Ces croyances incluent les jugements des apprenants sur leurs capacités à réaliser une tâche (comme le sentiment d'efficacité personnelle, voir Bandura, 2003), les buts qu'ils poursuivent dans leur scolarité, leurs études ou leur formation (comme les buts de compétence, voir Dweck \& Leggett, 1988), leur perception de contrôle sur leurs performances et leur façon d'expliquer leurs succès et échecs (styles attributifs, voir Weiner, 1986), ou encore la valeur qu'ils attribuent aux tâches et aux sujets scolaires (Wigfield \& Eccles, 2002). Notons que les croyances motivationnelles sont interrogées au niveau d'un sujet (par exemple les mathématiques) par certains et au niveau d'une tâche d'apprentissage par d'autres (Boekaerts les nomme alors "appréciations »). Notre propos ne portera pas sur des définitions extensives des champs de la métacognition et des croyances motivationnelles, mais sur les articulations opérées tant théoriquement qu'empiriquement entre ces deux champs conceptuels.

\section{ARGUMENTS THÉORIQUES EN FAVEUR D'UNE INTÉGRATION CONCEPTUELLE DE LA MÉTACOGNITION ET DES CROYANCES MOTIVATIONNELLES}

\section{Critiques de la cognition froide : limites des modèles purement cognitifs de l'apprentissage scolaire}

Il y aura bientôt trente ans, Brown, Bransford, Ferrara et alii (1983) soulignaient déjà l'absence des facteurs non cognitifs dans les modèles de la cognition scolaire : " academic cognition is cold, in that the principal concern is with the knowledge and strategies necessary for efficiency, with little emphasis placed on emotional factors that might promote or impede that efficiency 5 " (Brown, Bransford, Ferrara et al., 1983, p. 78). Selon d'autres chercheurs, tels Tobias et Everson (1997), le changement paradigmatique ayant amené une orientation cognitive à la psychologie aurait déclenché une période intense de recherches vouées à la clarification des processus cognitifs impliqués dans le comportement humain. Cependant, alors que ce changement a largement permis d'approfondir notre compréhension des multiples activités humaines, ces progrès se seraient réalisés aux dépens d'une compréhension plus fine du rôle des variables affectives dans ces mêmes activités. L'analyse et la compréhension des relations entre variables affectives et variables cognitives auraient été particulièrement négligées. 
Les modèles purement cognitifs sont critiqués également par Pintrich, Marx et Boyle (1993), qui affirment que ces modèles ne peuvent pas expliquer de façon adéquate pourquoi les élèves qui semblent avoir les prérequis conceptuels dans une certaine matière n'activent pas forcément ces connaissances pour différentes tâches scolaires (il s'agit du problème dit de "savoir inerte " décrit par Bereiter \& Scardamalia, 1985). Bien que le problème du savoir inerte puisse être attribué à des facteurs purement cognitifs tels que le niveau d'automatisation, la qualité de l'encodage ou des activités métacognitives insuffisantes, il est probable que des facteurs motivationnels et contextuels jouent également un rôle. Ainsi, si les modèles purement cognitifs sont utiles pour des situations expérimentales, ils sont moins pertinents pour des situations de classe, dans lesquelles les élèves ont une perception largement subjective des tâches et adoptent des buts et objectifs divers dans leur travail scolaire. Bandura (2003) ajoute que si l'adjonction des compétences métacognitives a permis d'élargir la théorie cognitive durant les années soixante-dix et quatre-vingt, les processus motivationnels, affectifs et de référence à soi sont encore négligés, bien qu'ils jouent un rôle primordial dans le développement et le fonctionnement cognitif. Une différence importante existe, en effet, entre disposer de certaines connaissances et utiliser ces connaissances de manière effective. En d'autres termes, savoir comment s'y prendre dans une tâche n'est pas suffisant en soi pour réussir à accomplir cette tâche. Les échecs dans les performances intellectuelles proviennent en effet souvent du sentiment qu'une approche métacognitive représenterait un investissement trop coûteux ou prendrait trop de temps, et pas simplement d'un manque de connaissances (Bandura, 1986 ; Flavell, 1976). Dans leur modèle du développement de la métacognition, Borkowski, Milstead et Hale (1988) parlent à ce sujet d'un manque de connaissances générales des stratégies. Ceci implique la recherche de facteurs explicatifs qui se trouvent probablement dans les domaines affectifs et motivationnels. L'un des facteurs explicatifs majeurs serait le sentiment d'efficacité personnelle (Bandura, 2003 ; Borkowski, Milstead \& Hale, 1988). Celui-ci serait nécessaire aux individus pour appliquer ce qu'ils savent de manière cohérente et en persistant dans leurs intentions, ceci particulièrement lorsque la résolution de la tâche ne se déroule pas facilement et qu'un échec pourrait avoir des conséquences indésirables.

Depuis quelques années, il existe une forte prise de conscience du besoin d'une articulation des aspects cognitifs de l'apprentissage scolaire avec les facteurs motivationnels et affectifs. En effet, Dai et Sternberg (2004) affirment que réduire le fonctionnement et le développement intellectuel à des phénomènes principalement cognitifs, c'est-à-dire ne pas prendre en considération les aspects motivationnels et émotionnels, n'est de nos jours plus soutenable tant au niveau théorique qu'à la lumière des preuves empiriques. Ceci est particulièrement pertinent pour l'étude du fonctionnement intellectuel d'élèves, indépendamment du cursus suivi, dans des situations de classe, mais moins pour les études en laboratoire. Dans ce dernier cas, le chercheur instaure une situation autant que possible contrôlée et l'influence des facteurs affectifs et motivationnels peut ainsi être, du moins partiellement, neutralisée.

\section{L'intégration des croyances motivationnelles dans les théories métacognitives}

II est intéressant de constater que les initiateurs de l'étude de la métacognition avaient, dès les années soixante-dix, relevé l'interdépendance de la métacognition et de la motivation. En effet, selon Brown (1978), « because metacognition demands the ability to introspect about one's own performance and [...] self-evaluation of one's own performance cannot be objective-such self-interrogation is contamined 
by one's own feelings of competence-some previously separate areas of personality development are again of obvious relevance (e.g. fear of failure, need for achievement, external vs internal control, learned helplessness, and level of aspiration) ${ }^{6}$ " (Brown, 1978, p. 80-81). L'autre parrain de la métacognition, Flavell (1987), mentionne parmi les facteurs de développement de la métacognition la croissance du sens de soi en tant qu'agent cognitif (la notion d'agentivité7, voir aussi Bandura, 2003), c'est-à-dire la prise de conscience progressive que l'individu est responsable de sa propre activité cognitive. Ceci est lié au développement de la perception de contrôle, prérequis nécessaire à l'engagement cognitif (Skinner, Zimmer-Gembeck \& Connell, 1998). L'un des liens les plus évidents est que la perception de contrôle serait promotrice des jugements et sentiments métacognitifs ainsi que des stratégies métacognitives lors des opérations cognitives. En effet, ce n'est qu'à la condition que l'élève perçoive un contrôle sur les résultats d'une tâche qu'il s'efforcera d'évaluer le déroulement de la résolution de cette tâche et de réguler son fonctionnement cognitif.

D'autres théoriciens de la métacognition, Paris et Winograd (1990), décrivent la présence de deux composantes dans leur définition de la métacognition : l'autoévaluation (self-appraisal) et l'auto-direction (self-management). L'auto-évaluation comprend les réflexions personnelles des individus au sujet non seulement de l'état de leurs connaissances et capacités mais aussi au sujet de leurs états affectifs, motivationnels et de leurs caractéristiques en tant qu'apprenants. De cette manière, les croyances motivationnelles sont intégrées dans la définition même de la métacognition. L'auto-direction concerne la métacognition en action, soit les processus mentaux qui permettent d'orchestrer les aspects de la résolution de problème (nous utilisons dans cette note de synthèse les termes "stratégies métacognitives " pour désigner ces processus). Ces deux composantes suggèrent que l'apprenant est nécessairement impliqué activement dans l'orchestration de la construction de ses connaissances. Notons que le concept d'expérience métacognitive, introduit par Flavell en 1979, incluait déjà des sentiments relatifs par exemple à la difficulté ou des sentiments ressentis par l'élève durant les opérations cognitives. S'y trouvait déjà un mélange de jugements cognitifs (par exemple le jugement de compréhension) et motivationnels ou affectifs (par exemple le sentiment que l'on ne réussira pas à résoudre la tâche). Dans leur texte de 1975, Kreutzer, Leonard et Flavell parlent de "mnemonic sensations ", concept qui inclut le sentiment qu'une information est nouvelle, qu'une autre a déjà été acquise mais n'est pas immédiatement accessible et qu'une troisième est "sur le bout de la langue " (on the tip of one's tongue). Ces sentiments ont été étudiés plus tard par Nelson sous les termes de « jugements métacognitifs » (Nelson \& Narens, 1990). Efklides et ses collaborateurs (Efklides, 2001, 2006) ont poursuivi les recherches dans ce domaine et étudié tant les antécédents que l'influence de nombreuses expériences métacognitives à la jonction de la cognition et de la motivation ou de l'affect. Autant Flavell qu'Efklides argumentent que des sentiments tels que le sentiment de difficulté, la satisfaction ou encore le sentiment qu'une tâche est plaisante ont un caractère métacognitif car ils constituent des jugements et sentiments sur l'activité cognitive en cours. Au contraire, beaucoup de chercheurs ne considèrent pas ces sentiments comme métacognitifs et les traitent comme des manifestations de la perception ou des appréciations de la tâche (appraisals, voir Boekaerts, 1999). Malgré ces différentes relations entre aspects motivationnels et aspects métacognitifs, les théories de la métacognition se sont développées de façon largement indépendantes des théories psychologiques de la motivation à apprendre. Ce n'est que récemment, avec l'apparition des modèles de l'apprentissage autorégulé (self-regulated learning), que les chercheurs ont adopté de façon effective une perspective plus large de l'apprenant incluant tant des aspects métacognitifs que motivationnels. 
Il y a une vingtaine d'années, Weinert (1987) avait lui aussi demandé une intégration des théories du domaine de la motivation aux théories du domaine de la cognition, car selon lui ces deux traditions avaient été jusque-là largement indépendantes. Un essai d'intégration impliquerait, selon Weinert, de lier les concepts suivants : d'un côté des concepts sur les connaissances de soi, les attentes de performance (c'est-à-dire les connaissances métacognitives) et le monitorage de ses propres actions (les jugements, les sentiments et les stratégies métacognitives), tels que définis dans la littérature métacognitive, et d'un autre côté des concepts tels que la perception de ses propres capacités (par exemple le sentiment d'efficacité personnelle), les attentes de succès, la peur de l'échec, ou encore les attributions causales traditionnellement traitées dans les théories de la motivation.

Plusieurs recoupements entre les deux domaines sont relevés par Weinert (1987). Par exemple, les attributions causales sont en lien avec la prédiction des performances futures (Weiner, 1986), tant dans la tradition métacognitive que dans les modèles motivationnels. Dans les théories métacognitives, cette prédiction est incluse dans les métaconnaissances sur soi, alors que dans les théories motivationnelles, elle est désignée par le terme "attente de succès ". Les mêmes concepts seraient ainsi interprétés de façon différente en fonction des théories. Pintrich, Wolters et Baxter (2000) proposent à ce sujet d'interpréter les métaconnaissances sur soi (à l'exception des connaissances sur le fonctionnement de la cognition humaine en général) en termes motivationnels plutôt que métacognitifs. Ceci concerne par exemple le concept de soi scolaire, le style attributif ou encore le sentiment d'efficacité personnelle. En effet, les connaissances métacognitives par rapport à soi incluent dans leur définition originelle (Flavell, 1987) des croyances sur soi en termes de différences interindividuelles (par exemple penser que son camarade est plus doué dans un certain domaine que soi-même) et intraindividuelles (penser de soi-même que l'on est plus doué pour l'orthographe que pour l'arithmétique), qui correspondent à des construits comme le concept de soi. La métacognition est selon Mandelman, Tan, Kornilov et alii (2010) l'une des sources à l'origine du concept de soi. Ces chercheurs postulent que le concept de soi scolaire ne serait pas uniquement formé sur la synthèse de facteurs externes à l'élève (c'est-à-dire l'environnement et l'entourage), mais également grâce à une source interne à l'élève, c'est-à-dire grâce à ses connaissances métacognitives au sujet de ses forces et faiblesses. Dans le même ordre d'idées, Markus et Wurf (1987) avaient décrit l'auto-évaluation comme un processus à la source du concept de soi. Les individus réalisent des inférences aux sujets de leurs attitudes et dispositions lorsqu'ils observent leurs propres conduites. L'auto-évaluation est combinée aux résultats des processus de comparaison sociale pour former ou ajuster le concept de soi. Par ailleurs, dans la taxonomie des connaissances cognitives de Kluwe (1982), il est fait référence aux connaissances des différences intra-individuelles au sujet des forces et faiblesses de l'individu en fonction des domaines, ce qui réfère au sentiment d'efficacité ou au concept de soi. En outre, Weinert (1987) souligne que si certaines variables des deux champs concernés sont identiques, leur fonction diffère. Par exemple, les jugements de la difficulté d'une tâche ou l'évaluation des résultats de l'action produisent des réactions émotionnelles et leurs conséquences sont prédites par les théories du type motivationnel. Par contre, dans le cadre des recherches métacognitives, ces variables sont liées aux connaissances métacognitives sur soi-même, sur la tâche et sur les stratégies (Flavell, 1987).

Weinert (1987) soulève cependant quelques problèmes relatifs à cette intégration. L'une des difficultés pour intégrer ces deux domaines, en prenant pour exemple les recherches sur la mémoire et la métamémoire, est la suivante : I'utilisation de types de tâche différents pour l'étude de la mémoire, de la métamémoire et de la 
motivation mène inévitablement à des conclusions différentes. Ceci a été le cas pour l'importance des connaissances spécifiques à un domaine, les capacités procédurales et les croyances motivationnelles pour la prédiction ou l'explication des différences individuelles dans l'apprentissage et la performance. De plus, toujours selon Weinert, le rôle des processus motivationnels a rarement été considéré car il n'y a pas eu d'effort systématique pour étudier la façon dont les personnes ayant des caractéristiques motivationnelles spécifiques se comportent face à différentes tâches dans des situations qu'elles considèrent importantes. II existe toutefois suffisamment de recouvrement entre les tâches et objets dans les champs de la cognition, métacognition et motivation pour développer des recherches intégrant les trois domaines. Par exemple, Borkowski et ses collaborateurs (Borkowski, Chan \& Muthukrishna, 2000) ont intégré plusieurs variables du domaine de la motivation dans leur modèle théorique du développement de la métacognition (voir la description détaillée de leur modèle ci-après).

En outre, les cognitions faisant référence à soi-même (en particulier la perception du contrôle et le sentiment d'efficacité personnelle) sont considérées par plusieurs chercheurs (notamment Bandura, 1986 ; Skinner, 1996 ; Weiner, 1986) comme de forts stimulateurs ou au contraire inhibiteurs du comportement en général et de l'apprentissage en particulier. Elles influencent l'évaluation par l'élève et ses autorégulations cognitives et motivationnelles en donnant du sens et de la valeur aux tâches et situations d'apprentissage (Boekaerts, 1996). Ainsi, se percevoir comme un élève stratégique et efficace dans l'utilisation de stratégies est considéré comme l'une des principales sources de motivation à apprendre (Pressley, Borkowski \& Schneider, 1987).

Le concept des "sois possibles " se réfère aux idées de l'individu sur ce qu'il pourrait devenir dans le futur, ce qu'il désirerait devenir et ce qu'il redouterait de devenir. Il s'agit en quelque sorte du concept de soi considérant le soi non seulement dans le présent mais également dans le passé et le futur. Les sois possibles servent de cadre pour indiquer à l'individu ce qui est envisageable qu'il accomplisse et pour guider son comportement vers ceci. Selon Day, Borkowski, Dietmeyer et alii (1992), les sois possibles pourraient également jouer le rôle de facilitateur ou être à l'origine de l'autorégulation (stratégies métacognitives). En effet, les sois possibles sont des buts à long terme et ils sont nécessaires pour donner forme ou diriger la sélection de stratégies, la planification ou plus généralement l'autorégulation (Markus, Cross \& Wurf, 1990). De cette manière, les sois possibles auraient un effet « énergisant » (c'est-à-dire promoteur) sur les stratégies métacognitives plutôt que sur des comportements stratégiques d'ordre inférieur. Les mécanismes ou processus précis ne sont toutefois pas détaillés par Day, Borkowski, Dietmeyer et alii (1992). En outre, les sois possibles ne contiendraient pas seulement des informations sur les buts finaux mais aussi sur la ou les manières de les atteindre. Si ces informations concernent l'efficacité de différentes stratégies, elles sont par conséquent de type métacognitif (ce sont des connaissances métacognitives). Cette théorie des sois possibles permet donc de lier le soi au fonctionnement métacognitif de l'individu. Elle ignore toutefois l'effet négatif que pourrait avoir un soi possible redouté ou dévalorisé sur l'autorégulation. Finalement, relevons que ces hypothèses théoriques n'ont à notre connaissance pas été testées empiriquement. En plus des multiples intégrations rapportées ci-dessus, les études portant sur la métamémoire ont récemment commencé à considérer le rôle des croyances motivationnelles dans le fonctionnement mnésique. 


\section{Extension des jugements métacognitifs considérés dans l'étude de la métamémoire}

Les chercheurs en psychologie cognitive, qui se réfèrent généralement au modèle de Nelson et Narens (1990) pour l'étude de la métamémoire, commencent également à s'intéresser au rôle des facteurs affectifs et motivationnels dans le fonctionnement métacognitif. L'un des présupposés du cadre théorique de Nelson et Narens (1990) est que les expériences subjectives (c'est-à-dire les jugements et sentiments métacognitifs) ne sont pas un épiphénomène, mais qu'elles modifient et guident les processus contrôlés cognitivement, ainsi que le comportement (Koriat, 2002 ; Son \& Schwartz, 2002). Les pensées et les sentiments subjectifs jouent ainsi un rôle causal prépondérant dans la régulation des processus cognitifs (Nelson, 1996). Ceci implique de considérer, dans les analyses métacognitives, des aspects affectifs et motivationnels, car ils font partie des expériences subjectives et ne doivent par conséquent pas être négligés ou ignorés : "Clearly, one's general beliefs about memory and the variables that affect it should contribute to one's metacognitive judgments in any given situation ${ }^{8}$ " (Koriat, 2002, p. 267).

Une ébauche d'intégration des facteurs motivationnels dans le modèle de Nelson et Narens (1990) a été proposée par Son et Metcalfe (2000), qui ajoutent aux habituels jugements métacognitifs étudiés en psychologie cognitive (ease-of-learning ou jugement de facilité de l'apprentissage, judgment-of-learning ou jugement de l'apprentissage, feeling-of-knowing ou setiment de connaître et confidence judgment ou jugement de confiance) un jugement relatif à l'intérêt de la tâche. Ce jugement, combiné au jugement de facilité de l'apprentissage, est d'ailleurs prédictif du temps d'étude alloué aux items dans une tâche d'apprentissage de biographies de personnes célèbres. En effet, plus une biographie est jugée intéressante et facile à apprendre, plus les participants allouent du temps à l'étude de cette biographie. De ces résultats, Son et Metcalfe (2000) concluent que la relation entre jugements et sentiments métacognitifs et stratégies métacognitives serait influencée par de nombreux facteurs encore peu considérés par les recherches. Selon nous, les facteurs négligés les plus importants sont les variables motivationnelles et affectives. D'autres chercheurs, tels que Schwartz et Perfect (2002), citent le concept de memory self-efficacy (sentiment d'efficacité mnésique) mais ils ne l'incluent pas parmi les jugements métacognitifs, alors qu'il est très proche du ease-of-learning, ce dernier étant un jugement de la facilité d'apprentissage d'une certaine tâche. En effet, sentiment d'efficacité personnelle et jugement de la difficulté d'une tâche sont souvent impossibles à distinguer empiriquement, comme cela a été observé notamment dans l'étude de Crombach, Boekaerts et Voeten (2003), ainsi que dans nos propres travaux (voir par exemple Berger, 2009).

Dans le même ordre d'idées, Hacker et Bol (2004) soulignent l'importance de considérer le construit du sentiment d'efficacité personnelle dans la compréhension du fonctionnement de la métamémoire. Selon ces auteurs, les jugements sur ses propres connaissances et processus cognitifs sont influencés par des facteurs tels que : le sentiment qu'une personne peut se souvenir d'une connaissance, la compétence d'évaluer correctement l'adéquation de cette connaissance à la réponse aux tâches dans lesquelles elle est engagée, ainsi que les croyances sur soi-même en tant qu'individu capable de mémorisation. En outre, Hacker et Bol (2004) postulent que le style attributif joue un rôle dans les jugements métacognitifs. Ces chercheurs constatent que les élèves scolairement faibles sont généralement à la fois moins précis et trop confiants dans leurs jugements métacognitifs par comparaison à des élèves scolairement doués, ceux-ci étant trop peu confiants. Selon Hacker et Bol (2004), cette différence pourrait être due au style attributif. Leur hypothèse est que les élèves scolairement faibles surestiment régulièrement leurs performances et 
attribuent leurs échecs à des facteurs incontrôlables afin de protéger leur estime d'eux-mêmes. En d'autres termes, ils s'évaluent de manière à conserver une image d'eux-mêmes d'élèves relativement bons en comparaison aux autres. Ces auteurs soulignent également que ce n'est pas seulement le soi privé qui ne doit pas être touché ou détérioré par les jugements métacognitifs, mais que le soi public se doit aussi d'être protégé9.

\section{Expériences métacognitives et attributions causales}

Metallidou et Efklides (2001) ont étudié les effets des expériences métacognitives sur les attributions causales des élèves suite à la résolution d'un problème mathématique. Elles s'interrogent sur le rôle de l'expérience subjective de l'élève, sous la forme d'expériences métacognitives, dans le processus attributif. Ces chercheuses postulent que les attributions causales seraient basées sur l'utilisation d'expériences métacognitives et de croyances plus générales liées notamment au rôle de l'effort et au rôle des aptitudes dans le succès (ces croyances ont été abordées sous les termes de "perception de contrôle »). Metallidou et Efklides (2001) relèvent que les théories actuelles recherchent les origines du processus attributif dans des facteurs indépendants de la tâche concernée par l'attribution, alors qu'une des attributions majeures, à savoir la difficulté de la tâche, se réfère directement à la conception subjective de la tâche ou des attributs de la situation. Ceci signifie que les expériences métacognitives spécifiques à la tâche participeraient au mécanisme de production d'une attribution causale. Les attributions causales à l'effort seraient également influencées de cette façon, puisque l'estimation par l'élève de la quantité d'efforts qu'il a fourni est un indice qui semble pertinent pour prédire une attribution causale du résultat à l'effort. Ces auteures postulent également que les sentiments de confiance et de satisfaction seraient liés à l'attribution causale des succès et échecs aux capacités des élèves, étant donné que ces sentiments informent l'élève à propos de l'atteinte de ses buts (c'est-à-dire le degré de réussite dans la tâche). Tous ces postulats justifient l'hypothèse d'un rôle des expériences métacognitives dans le processus attributif. Par des analyses de cheminement, Metallidou et Efklides (2001) montrent que les expériences métacognitives jouent effectivement un rôle médiateur dans la formation des attributions causales. Si les croyances relatives au contrôle expliquent les attributions causales, leur impact est cependant largement indirect, car les expériences métacognitives jouent un rôle médiateur. La façon habituelle des élèves d'expliquer leurs résultats est activée dans chaque nouvelle situation d'accomplissement et influence leur attitude envers la situation (dans cette étude, il s'agit de la perception du problème comme plus ou moins plaisant et de l'intention de le résoudre). L'unique influence directe des croyances de contrôle sur les attributions causales concerne les attributions à la chance, qui dépendent logiquement en partie des croyances que la chance est responsable du succès scolaire.

Les effets des expériences métacognitives évaluées avant la résolution de la tâche sur les attributions causales sont limités, alors que les expériences métacognitives réalisées pendant et après la résolution ont des effets plus prononcés. Les jugements de confiance affectent directement les attributions aux capacités, c'est-à-dire que la confiance est interprétée comme un indicateur de la capacité de la personne à répondre aux demandes de la situation. Le sentiment de difficulté est directement lié aux attributions de la réussite ou de l'échec à la difficulté de la tâche. Toutefois, il n'y a pas de correspondance une à une des expériences métacognitives avec les attributions, puisque plusieurs expériences métacognitives peuvent contribuer à la formation d'une unique attribution causale. II semble ainsi 
que l'expérience subjective (les expériences métacognitives) offre un feedback qui permet à l'élève de se juger par rapport à la tâche en cours en comparant cette expérience avec ce qu'il pense (sur la base de son expérience scolaire) des causes de succès ou d'échec dans des situations similaires. Cet exemple illustre l'intégration de construits motivationnels et métacognitifs dans la compréhension du fonctionnement psychologique de l'élève. Toutefois, tous les chercheurs ne sont pas en accord sur ce type d'intégration ainsi que sur leur utilité.

\section{Limites dans l'articulation théorique de la métacognition et des croyances motivationnelles}

Les différentes intégrations de variables motivationnelles dans les théories métacognitives sont critiquées principalement par Boekaerts $(1997,1999)$ car elles posent des problèmes conceptuels. En particulier le fait que les nomenclatures ou les terminologies diffèrent. En effet, l'élargissement du construit de connaissances métacognitives à des aspects de croyances motivationnelles (par exemple le sentiment d'efficacité personnelle ou les buts de compétence), à des aspects de la théorie de l'esprit de l'élève, à des théories sur soi, aux théories de l'apprentissage ou même à l'environnement d'apprentissage (Thomas, 2003) conduit à un accroissement du flou conceptuel. Or les théories métacognitives sont déjà fortement critiquées pour la trop grande multiplicité des termes utilisés pour désigner des construits similaires. Par conséquent, Boekaerts (1999) propose, comme Pintrich, Wolters et Baxter (2000), de limiter les métaconnaissances à des aspects liés aux stratégies et aux tâches. Le second argument de Boekaerts (1997) est que les perspectives des deux traditions de recherche sont différentes et qu'elles doivent le rester afin de garder leurs pouvoirs explicatifs respectifs. En effet, les recherches dans le champ de la métacognition sont centrées sur les compétences cognitives et les connaissances relatives aux tâches (notamment les jugements des élèves relatifs aux stratégies qu'ils utilisent ou à la difficulté de la tâche), alors que les recherches du domaine de la motivation seraient centrées sur les caractéristiques du soi (notamment la perception et l'interprétation des activités en fonction de leurs besoins psychologiques). II existerait de plus en plus de preuves que les croyances motivationnelles sont des entités distinctes qui agissent sur l'activité d'apprentissage mais également sur des activités concurrentes qui passent par l'esprit de l'élève. En cas de traitement de ces croyances comme connaissances métacognitives, leur pouvoir explicatif risque d'être réduit. Les chercheurs dans le cadre sociocognitif préfèrent ainsi parler d'apprentissage autorégulé lorsqu'il s'agit de décrire et prédire les effets des croyances motivationnelles et de la métacognition sur l'apprentissage. Les deux domaines sont considérés comme distincts mais ils sont articulés dans un même cadre théorique, comme le propose par exemple Boekaerts $(1996,1997)$ ou Pintrich (2004). Nous allons maintenant nous pencher sur ces modèles par la description de deux d'entre eux considérés comme révélateurs de l'intégration théorique entre métacognition et motivation.

\section{LES MODÈLES D'APPRENTISSAGE AUTORÉGULÉ}

Les deux modèles de l'apprentissage autorégulé que nous présentons ont été élaborés par des chercheurs nord-américains (Borkowski, Chan \& Muthukrishna, 2000 ; Pintrich, 2000, 2004). Selon Pintrich (2004), cette conception est dérivée de l'approche dite du traitement de l'information (information processing approach), suite aux critiques sur les limites de cette dernière, et adopte une perspective plus 
largement inclusive. En effet, au contraire des modèles inspirés de l'approche du traitement de l'information, la conception de l'apprentissage autorégulé traite l'apprentissage selon des facteurs non seulement cognitifs, mais aussi motivationnels et affectifs, ainsi que sociaux et contextuels. Par conséquent, ces modèles offrent une description plus riche de l'apprentissage et de la motivation des élèves que ce n'était le cas dans les modèles du traitement de l'information. Nous présentons ici les modèles heuristiques développés par Borkowski et par Pintrich, que nous considérons comme étant parmi les plus pertinents par rapport à l'articulation entre métacognition et motivation. Signalons que de nombreux autres modèles de l'apprentissage autorégulé, impliquant différents construits et différentes perspectives, ont été développés depuis les années quatre-vingt-dix (par exemple Boekaerts, 1997 ; Bouffard-Bouchard, Parent \& Larivée, 1990 ; De Corte, Verschaffel \& Op't Eynde, 2000 ; Laveault, Leblanc \& Leroux, 1999 ; Winne \& Hadwin, 1998 ; Zimmerman, 2000 ; voir Cosnefroy, 2011, pour une revue en français des modèles nordaméricains majeurs), preuve d'un enthousiasme certain pour ce champ de recherche.

\section{Le modèle du développement métacognitif de Borkowski}

Les travaux de Borkowski et ses collaborateurs (voir par exexemple Borkowski, Chan \& Muthukrishna, 2000 ; Borkowski, Johnston \& Reid, 1987 ; Borkowski, Reid \& Kurtz, 1984 ; Borkowski, Weyhing \& Carr, 1988 ; Carr, Borkowski \& Maxwell, 1991 ; Kurtz \& Borkowski, 1987) sur le rôle des variables motivationnelles dans le fonctionnement et le développement métacognitif ont été essentiels dans l'élargissement de la théorie métacognitive. Leur modèle de la métacognition est d'une part développemental en ce qu'il décrit l'évolution des composantes de la métacognition en lien avec les croyances motivationnelles; d'autre part, nous considérons ce modèle comme un modèle de l'apprentissage autorégulé, bien que Borkowski lui-même ne le qualifie pas ainsi. Ce modèle partage en effet les postulats qui sont selon Pintrich à la base des modèles d'autorégulation (voir ci-après pour une description de ces postulats). Soulignons, toutefois, que les domaines d'autorégulation sont limités dans le modèle de Borkowski à la cognition et à la motivation.

Borkowski et ses collaborateurs (Borkowski, Chan \& Muthukrishna, 2000) introduisent, dans leur théorie de la métacognition, le concept d'« états personnels et motivationnels " (personal-motivational states), en y incluant notamment le style attributif, la motivation d'accomplissement ainsi que la motivation intrinsèque. Leur modèle complet de la métacognition comprend, de plus, des variables relatives à la connaissance de soi (self-system), en particulier l'estime de soi, le sentiment d'efficacité personnelle et les sois possibles. Borkowski a élaboré également un modèle d'intervention se centrant principalement sur l'amélioration des stratégies d'apprentissage. Ces dernières sont non seulement indispensables pour un apprentissage efficace, mais elles servent également de fondement à l'entraînement explicite de ce que Borkowski nomme les " processus exécutifs " (jugements, sentiments et stratégies métacognitives), à la modification du style attributif ainsi qu'à l'amélioration du sentiment d'efficacité personnelle. II décrit schématiquement le développement métacognitif qui s'opère chez les élèves qui sont confrontés à cet enseignement de la façon suivante : tout d'abord, l'élève développe ses connaissances des stratégies spécifiques (specific strategy knowledge), qui incluent leur efficacité, leur étendue d'application, ainsi que leur utilisation avec différents types de tâches (autrement dit des connaissances métacognitives). Les processus exécutifs apparaissent ensuite avec le développement de la capacité à sélectionner les stratégies appropriées et à combler ses déficits de connaissances en utilisant le monitorage ; ces processus servent à analyser la tâche et à choisir une stratégie appropriée puis, 
pendant l'apprentissage, ils sont utiles pour le monitorage et le contrôle. Ensuite l'amélioration des processus stratégiques et exécutifs permet à l'élève de prendre conscience de l'utilité et de l'importance d'un comportement stratégique. Ceci implique que les connaissances d'ordre général sur les stratégies s'accroissent et que le sentiment d'efficacité personnelle se développe. L'élève apprend à attribuer le succès ou l'échec à l'effort qu'il a fourni dans le déploiement des stratégies plutôt qu'à des facteurs incontrôlables tels que la difficulté de la tâche (développement de la perception de contrôle). L'élève reçoit ou infère, après la plupart de ses activités cognitives, un feedback à propos de la qualité de sa performance et de ses déterminants. Ce feedback permet de modifier les états motivationnels qui influencent à leur tour notamment les stratégies métacognitives. Le modèle intègre donc des activités cognitives (sous la forme d'utilisation des stratégies) avec leurs causes et leurs conséquences motivationnelles. La connaissance de soi joue quant à elle un rôle de stimulateur pour le système métacognitif global.

Borkowski, Chan et Muthukrishna (2000) postulent, sur la base de leurs recherches empiriques (Borkowski, Milstead \& Hale, 1988 ; Carr, Borkowski \& Maxwell, 1991 ; Reid \& Borkowski, 1987), que les attributions causales, au même titre que la motivation intrinsèque par exemple, influencent le fonctionnement exécutif de l'élève. Des modifications à long terme du comportement stratégique d'un élève seraient dépendantes de relations complexes entre les métaconnaissances, le fonctionnement exécutif et les croyances motivationnelles, dont les attributions causales. Les attributions causales des résultats à l'utilisation de stratégies en particulier joueraient un rôle moteur important dans l'utilisation des jugements et sentiments métacognitifs et des stratégies métacognitives, ainsi que plus globalement dans le développement de la métacognition.

Selon Borkowski, les élèves qui croient que les efforts qu'ils fournissent dans l'utilisation de stratégies vont leur permettre de réussir les tâches seront probablement actifs dans la sélection des stratégies, le monitorage et l'utilisation de stratégies métacognitives, composantes du fonctionnement exécutif. La perception de contrôle personnel (attributions aux efforts et stratégies) est positivement liée aux connaissances et à l'utilisation des stratégies (voir par exemple Borkowski, Milstead \& Hale, 1988). Ce modèle théorique n'a pas été vérifié empiriquement dans sa globalité. Toutefois une étude avec des élèves en difficulté d'apprentissage a confirmé les liens entre une partie des variables motivationnelles et métacognitives (Carr, Borkowski \& Maxwell, 1991) considérées dans le modèle de Borkowski. Les auteurs ont mesuré le style attributif, l'estime de soi, les métaconnaissances relatives aux stratégies en lecture (notamment l'évaluation des composantes de la tâche, des capacités en lecture, des stratégies de planification pour faciliter la compréhension et des stratégies de régulation de la compréhension), ainsi que la performance en lecture. Ils ont montré par un modèle de cheminement que les attributions causales jouaient un rôle essentiel et influençaient de façon indirecte le développement des stratégies et des connaissances métacognitives sur la lecture. Ainsi, un style attributif inapproprié (c'est-à-dire la non-reconnaissance du rôle des efforts dans la réussite et l'échec) freine l'acquisition des stratégies et des métaconnaissances puisque les élèves avec un style attributif externe n'ont pas de raison d'utiliser des stratégies dont ils pensent qu'elles ne les aideront pas à apprendre. De ce modèle et de ces études, nous retenons la complexité de relations entre les aspects motivationnels et affectifs et les aspects cognitifs et métacognitifs au cours du développement : si les croyances motivationnelles exercent un rôle promoteur ou au contraire restrictif dans la mobilisation et le développement des processus métacognitifs, l'application de stratégies influence, à l'opposé, les croyances motivationnelles, en particulier le sentiment d'efficacité personnelle. La causalité est ainsi réciproque. 


\section{Le modèle de l'apprentissage autorégulé de Pintrich}

\section{Présentation du modèle}

Selon Pintrich $(2000,2004)$, les modèles de l'apprentissage autorégulé partagent quatre postulats :

- les apprenants sont des participants actifs dans le processus d'apprentissage. Ils construisent leurs propres significations, buts et stratégies à partir des informations disponibles tant dans l'environnement que dans leur propre esprit. Ainsi, ces théories de l'apprentissage autorégulé postulent que l'apprentissage est de nature constructiviste ${ }^{10}$;

- les apprenants peuvent potentiellement évaluer, contrôler et réguler certains aspects de leur propre cognition, motivation et comportement ainsi que certaines caractéristiques de leur environnement. Ceci ne signifie pas pour autant qu'ils le fassent de manière continue ou régulière, étant donné les contraintes, notamment développementales, mais cela signifie qu'il s'agit uniquement d'une possibilité ;

- l'apprenant a des buts, des critères ou des standards avec lesquels il réalise des comparaisons afin d'évaluer si le processus d'apprentissage doit continuer de la même façon ou si des changements sont nécessaires ;

- les activités d'autorégulation jouent le rôle de médiateur entre les caractéristiques personnelles et contextuelles et la performance effective. Ceci signifie que l'autorégulation, dans chacun des domaines cognitif, motivationnel et affectif, comportemental et social ou contextuel, joue un rôle-clé.

Les théories de l'apprentissage autorégulé qui se sont développées récemment conçoivent celui-ci comme un processus actif et constructif par lequel les apprenants établissent des buts pour leur apprentissage et essaient de guider, réguler et contrôler leurs cognitions, motivations et comportements, orientés et contraints par leurs buts et les caractéristiques de l'environnement (Pintrich, 2000). L'élève qui autorégule parfaitement son apprentissage se perçoit comme agent de ses comportements, pense que l'apprentissage est un processus proactif, se motive et utilise des stratégies qui lui permettent d'obtenir les résultats scolaires qu'il souhaite. Parmi ces modèles théoriques, celui de Pintrich (décrit dans Pintrich, 2000, 2004 ; Pintrich \& Zusho, 2002) nous intéresse en particulier car il offre une description large des croyances motivationnelles et des composantes métacognitives en jeu lors de différentes phases d'un apprentissage. Ce modèle heuristique, présenté dans le tableau 1, a pour objectif de classer et d'analyser les processus de régulation selon une séquence générale théoriquement suivie dans la résolution d'une tâche.

Ces phases ne sont pas forcément ordonnées comme dans la présentation du modèle : elles peuvent être réalisées dans un ordre différent, être simultanées ou encore interagir de façon dynamique, ce qui produit des interactions complexes entre variables. Par ailleurs, ces phases correspondent approximativement aux «étapes » de stratégies métacognitives telles que définies généralement dans la littérature métacognitive (anticipation, planification et vérification). Seule la dernière phase, réactions et réflexion, ne rentre pas dans cette correspondance. Ces phases sont, en outre, caractéristiques des processus considérés dans les modèles de l'apprentissage autorégulé (par exemple elles sont proches de celles décrites dans le modèle de Zimmerman, 2000). Ces quatre phases sont chacune associées aux quatre domaines de régulation envisagés par Pintrich $(2000,2004)$ : cognition, motivation et affect, comportement et contexte. Dans chacun de ces quatre domaines, les apprenants peuvent exercer une certaine régulation. Nous nous attacherons à décrire dans le présent texte les deux premiers domaines (cognition et motivation 
Tableau 1. Les phases et domaines de l'apprentissage autorégulé adapté de Pintrich (2000)

\begin{tabular}{|c|c|c|c|c|}
\hline \multirow[b]{2}{*}{ Phases } & \multicolumn{4}{|c|}{ Domaines de régulation } \\
\hline & Cognition & $\begin{array}{l}\text { Motivation } \\
\text { et affect }\end{array}$ & Comportement & Contexte \\
\hline $\begin{array}{l}\text { Phase 1: } \\
\text { anticipation, } \\
\text { planification } \\
\text { et activation }\end{array}$ & $\begin{array}{l}\text { Établissement } \\
\text { d'un but } \\
\text { spécifique } \\
\text { Activation des } \\
\text { connaissances } \\
\text { antérieures } \\
\text { Activation des } \\
\text { connaissances } \\
\text { métacognitives }\end{array}$ & $\begin{array}{l}\text { Adoption } \\
\text { de buts de } \\
\text { compétence } \\
\text { Jugements } \\
\text { de sentiment } \\
\text { d'efficacité } \\
\text { Perception de } \\
\text { la difficulté de } \\
\text { la tâche } \\
\text { Activation de } \\
\text { la valeur de la } \\
\text { tâche }\end{array}$ & $\begin{array}{l}\text { Planification } \\
\text { du temps et de } \\
\text { l'effort } \\
\text { Planification } \\
\text { de l'auto- } \\
\text { observation du } \\
\text { comportement }\end{array}$ & $\begin{array}{l}\text { Perceptions de } \\
\text { la tâche } \\
\text { Perceptions du } \\
\text { contexte }\end{array}$ \\
\hline $\begin{array}{l}\text { Phase 2: } \\
\text { monitorage }\end{array}$ & $\begin{array}{l}\text { Conscience } \\
\text { métacognitive et } \\
\text { monitorage de la } \\
\text { cognition }\end{array}$ & $\begin{array}{l}\text { Conscience et } \\
\text { monitorage de } \\
\text { la motivation et } \\
\text { de l'affect }\end{array}$ & $\begin{array}{l}\text { Conscience } \\
\text { et monitorage } \\
\text { de l'effort, de } \\
\text { l'utilisation du } \\
\text { temps, du besoin } \\
\text { d'aide } \\
\text { Observation } \\
\text { de son propre } \\
\text { comportement }\end{array}$ & $\begin{array}{l}\text { Monitorage des } \\
\text { changements } \\
\text { de tâche et des } \\
\text { conditions du } \\
\text { contexte }\end{array}$ \\
\hline $\begin{array}{l}\text { Phase } 3 \text { : } \\
\text { contrôle }\end{array}$ & $\begin{array}{l}\text { Sélection et } \\
\text { adaptation } \\
\text { des stratégies } \\
\text { cognitives pour } \\
\text { l'apprentissage } \\
\text { et la pensée }\end{array}$ & $\begin{array}{l}\text { Sélection et } \\
\text { adaptation des } \\
\text { stratégies pour } \\
\text { le management } \\
\text { de la motivation } \\
\text { et de l'affect }\end{array}$ & $\begin{array}{l}\text { Augmentation } \\
\text { ou diminution de } \\
\text { l'effort } \\
\text { Persistance, } \\
\text { abandon, } \\
\text { comportement } \\
\text { de recherche } \\
\text { d'aide }\end{array}$ & $\begin{array}{l}\text { Changement ou } \\
\text { renégociation } \\
\text { de la tâche } \\
\text { Changer ou } \\
\text { quitter le } \\
\text { contexte }\end{array}$ \\
\hline $\begin{array}{l}\text { Phase 4: } \\
\text { réactions } \\
\text { et réflexion }\end{array}$ & $\begin{array}{l}\text { Jugements } \\
\text { cognitifs } \\
\text { Attributions } \\
\text { causales }\end{array}$ & $\begin{array}{l}\text { Réactions } \\
\text { affectives } \\
\text { Attributions } \\
\text { causales }\end{array}$ & $\begin{array}{l}\text { Comportements } \\
\text { de choix }\end{array}$ & $\begin{array}{l}\text { Évaluation de la } \\
\text { tâche }\end{array}$ \\
\hline
\end{tabular}


et affect) en fonction de chaque phase, étant donné qu'il s'agit de l'objet de cette note de synthèse, alors que les deux autres domaines ne sont qu'indirectement abordés. Les quatre phases du modèle, ainsi que leur association avec les domaines cognitif et motivationnel ou affectif sont les suivantes.

\section{Phase d'anticipation, de planification et d'activation}

Cette phase concerne la planification, l'établissement d'un but ainsi que l'activation des perceptions et connaissances de la tâche, du contexte et de soi en relation à la tâche. Par rapport au domaine de la cognition, l'élève active ses connaissances antérieures de façon consciente (par exemple en se demandant ce qu'il connaît déjà sur le sujet concerné par la tâche) ou automatique. Il peut s'agir de connaissances sur des contenus ou de connaissances métacognitives sur la tâche ou sur les stratégies. Ceci lui permet de développer une représentation plus élaborée de la tâche. Pendant une longue période, la plupart des chercheurs ont considéré l'activation de la structure des connaissances nécessairement consciente. Toutefois chacun de nous a déjà fait l'expérience d'une pensée qui nous est venue à l'esprit sans que nous puissions dire pourquoi ou, selon une formulation scientifique, une partie de la mémoire à long terme est activée et transférée dans l'exécutif central (Baddeley \& Logie, 1999). Norman (1979) a parlé de "slips of the mind " (glissements de l'esprit) qui peuvent provoquer des erreurs ou déclencher d'autres actions dont nous ne nous rendons pas immédiatement compte. Selon Norman, de telles expériences ne sont pas explicables sans que nous postulions un "autonomous, subconscious processing, with intentions, past habits, thoughts and memories ${ }^{11}$ " (Norman, 1979, p. 4). En 1975, Bobrow et Norman avaient déjà proposé l'idée de "context-dependent descriptions » (descriptions dépendantes du contexte). Selon cette théorie, une description représente un schéma lié à un contexte spécifique. Si une personne se trouve dans ce contexte, le schéma est alors activé automatiquement. D'après Norma et ses collègues, un schéma est une structure organisée et composée de connaissances déclaratives et procédurales ("organized memory units ", voir Norman, 1979, p. 6). Chaque schéma a été développé dans un contexte (ou une situation) spécifique. II représente une copie mnésique de cette situation et de notre première réaction à la situation. II nous permet de réagir de manière suffisamment adéquate à une situation future dont la similitude avec la situation initiale est reconnue grâce aux descriptions du schéma. Une description d'un schéma consiste en un nombre limité de concepts pertinents pour la description de la situation dans lequel le schéma a été développé (le schéma initial). Un schéma peut être déclenché de manière consciente ou automatique, selon le niveau de complexité de la situation et le niveau de traitement (Craik \& Lockhart, 1972) de la situation initiale. L'un de nous a utilisé cette idée pour développer un modèle de l'apprentissage (Büchel \& Büchel, 2009) dans lequel l'apprentissage est défini comme l'intégration d'un nouveau savoir dans la mémoire à long terme (MLT), une partie plutôt passive de notre mémoire. Pour préparer une telle intégration, la MLT est pré-activée de manière automatique par le contexte dans lequel l'apprentissage a lieu. Cette activation peut être considérée comme une anticipation automatique. En outre, l'élève peut se fixer un but, qui varie potentiellement pendant l'exécution de la tâche. Nous ajouterons ici la formulation d'un plan d'action, que celui-ci soit sommaire ou élaboré. Ce plan peut être formulé sur la base d'habitudes automatisées (par conséquent l'élève n'en est pas forcément conscient) ou de façon très explicite (par exemple pour résoudre un problème de mathématiques complexe).

Par rapport au domaine de la motivation et de l'affect, l'élève réalise différents jugements, en particulier sur son sentiment d'efficacité personnelle, la difficulté perçue de la tâche, l'intérêt situationnel, la valeur de la tâche (importance, utilité, coût, voir Wigfield \& Eccles, 2002). De plus, il adopte des buts de compétence en lien 
avec ces jugements. Des sentiments ou émotions négatives peuvent également être activés, tels que l'anxiété ou la peur qui influencent subséquemment le processus d'apprentissage ainsi que sa régulation. Tous ces éléments motivationnels et affectifs peuvent potentiellement être régulés par l'élève et ainsi être modifiés durant ou après la réalisation de la tâche.

\section{Phase de monitorage}

Cette phase concerne divers aspects des processus de monitorage qui représentent la conscience métacognitive de différents aspects de soi, de la tâche ou du contexte. Par rapport au domaine de la cognition, l'élève réalise des jugements pendant l'exécution de la tâche (par exemple sur sa compréhension). Ces jugements ont en particulier été traités dans le modèle théorique de Nelson et Narens (1990), comme par exemple le jugement de confiance, cité ci-dessus. Par rapport au domaine de la motivation et de l'affect, il s'agit pour l'élève de prendre conscience de sa propre motivation et de ses affects. Afin de pouvoir les contrôler et les réguler, l'élève doit nécessairement en avoir conscience, ce qui n'est pas forcément le cas. Peu de recherches se sont intéressées à cet aspect de l'autorégulation (pour des exceptions, voir Cosnefroy, 2010 ; Wolters, 2003). Quant aux expériences métacognitives définies par Efklides (2001), elles se situeraient entre monitorage de la cognition et monitorage de la motivation et de l'affect selon le modèle de Pintrich (2000).

\section{Phase de contrôle et de régulation}

Cette phase concerne les efforts réalisés pour contrôler et réguler différents aspects relatifs à soi, à la tâche ou au contexte. Par rapport au domaine de la cognition, le contrôle et la régulation sont postulés comme étant fortement liés au monitorage, bien que les deux processus soient considérés comme distincts en théorie. L'un des aspects centraux est, selon Pintrich (2000), la sélection et l'utilisation de stratégies cognitives diverses pour la mémorisation, l'apprentissage, le raisonnement ou encore la résolution de problème. Ce qui justifie le qualificatif de métacognitif attribué à ces stratégies est la décision ou le choix de les utiliser ainsi que la décision d'arrêter de les utiliser ou d'en changer, de les appliquer plus précisément, etc. Par rapport au domaine de la motivation et de l'affect, l'élève peut recourir à de multiples stratégies pour contrôler sa motivation et ses émotions. Parmi ces stratégies, les plus courantes seraient : se parler à soi-même de manière positive ou encourageante ("Je sais que je peux y arriver "), se promettre une récompense suite à la réalisation de la tâche, ou encore se répéter les conséquences positives de son travail ("Grâce au temps que je passe à étudier, j'aurai de bonnes notes ", voir Wolters, 2003). Mais il existe également des stratégies pour se protéger en cas d'échec, c'est-à-dire ne pas toucher l'estime de soi scolaire. II s'agit notamment de diminuer de manière délibérée la valeur d'une tâche ou du domaine ou valoriser des domaines non scolaires, des stratégies dites " auto-handicapantes " (selfhandicapping) telles que la procrastination qui permet d'attribuer un mauvais résultat au manque d'efforts plutôt qu'au manque de capacités et ainsi protéger l'estime de soi.

\section{Phase de réactions et de réflexion}

Cette phase concerne différents types de réactions et de réflexions sur soi, la tâche ou le contexte. Par rapport au domaine de la cognition, l'élève réalise dans cette phase des jugements et évaluations relatifs à sa performance (des expériences métacognitives selon Efklides), à la façon dont il a résolu la tâche (par exemple l'efficacité des stratégies appliquées) et il développe des attributions causales de sa performance. Les élèves qui s'autorégulent fortement auraient tendance à évaluer 
systématiquement leurs performances au contraire des élèves dont l'autorégulation est faible (Zimmerman, 2000). Par ailleurs, une différence dans le style attributif est également observée : les bons autorégulateurs attribuent plus largement, en comparaison des faibles autorégulateurs, leurs performances à des facteurs sur lesquels ils exercent un contrôle (l'effort et les stratégies) plutôt qu'à des facteurs hors de leur contrôle (Borkowski, Milstead \& Hale, 1988).

Par rapport au domaine de la motivation et de l'affect, les élèves peuvent réagir émotionnellement à leurs résultats et les attribuer à une ou plusieurs causes. Leurs attributions causales peuvent être biaisées ou contrôlées afin notamment de maintenir leur estime de soi. Ces attributions causales ont des effets importants sur les émotions ressenties et influencent les attentes futures de succès ainsi que le sentiment d'efficacité personnelle de l'élève (Weiner, 1986). De cette manière, les réactions motivationnelles et affectives influencent les pensées dans la phase d'anticipation, planification et activation lors des prochaines tâches. II s'agit ainsi d'un processus dynamique et cyclique. De manière similaire au modèle de Borkowski, une causalité réciproque est postulée. Les croyances motivationnelles activées dans la phase d'anticipation et de planification influencent les processus métacognitifs durant les phases suivantes. Les pensées activées dans la phase de réactions et de réflexion, en particulier les jugements de confiance, affectent les croyances motivationnelles lors de la prochaine phase d'anticipation et de planification.

\section{Précisions sur le modèle}

Parmi les nombreuses variables associées à chaque phase, on retrouve les connaissances métacognitives, les jugements et sentiments métacognitifs, ainsi que la sélection et l'adaptation des stratégies cognitives. La phase d'anticipation et de planification lie des concepts tels que les métaconnaissances et l'activation de connaissances antérieures aux buts de compétence et autres croyances motivationnelles telles que le sentiment d'efficacité personnelle ou la valeur accordée à la tâche. Les phases de monitorage et de contrôle lient la régulation de la cognition pendant l'exécution de la tâche avec la régulation de la motivation. La phase finale, réactions et réflexion, contient les jugements et évaluations de l'élève par rapport à son exécution de la tâche, ce qui correspond notamment au processus attributif.

Bien entendu, tous les apprentissages scolaires ne sont pas autorégulés ou ne suivent pas ces phases, étant donné que de nombreux apprentissages sont réalisés de manière relativement implicite, tacite ou non intentionnelle sans nécessiter de recours à l'autorégulation (Pintrich, 2000). En outre, d'autres personnes (enseignants, parents ou pairs) régulent également l'apprentissage de l'élève dans chacun des domaines considérés (il s'agit alors d'hétéro-régulation), par exemple en lui demandant de réaliser une tâche d'une certaine façon ou à un certain moment. Notons que la recherche d'aide auprès d'un camarade, parent ou enseignant est, au contraire, considérée comme une stratégie d'autorégulation malgré l'intervention d'un tiers car cette stratégie est initiée par l'apprenant lui-même (Karabenick \& Berger, 2012).

Nous retenons de ce modèle que l'autorégulation est considérée dans un sens large et qu'elle n'est pas uniquement dirigée vers des éléments internes à l'élève, mais qu'elle concerne aussi des éléments externes (la régulation du contexte). Les concepts sont très nombreux et la plupart des études sont réalisées en considérant les régulations de la cognition et de la motivation et de l'affect. Les études s'intéressant également à la régulation du comportement et du contexte sont plus rares. Nous évoquons maintenant une revue sélective d'études empiriques s'étant intéressées aux relations entre les croyances motivationnelles et les divers aspects de la métacognition. 


\section{ÉTUDES EMPIRIQUES ARTICULANT CROYANCES MOTIVATIONNELLES ET MÉTACOGNITION}

Un certain nombre d'études empiriques réalisées depuis les années quatre-vingt ont permis de mettre au jour de nombreux liens entre construits motivationnels et construits métacognitifs. Nous passons en revue ci-dessous une partie de ces études empiriques en considérant successivement les relations avec les croyances motivationnelles suivantes : le sentiment d'efficacité personnelle, les buts de compétence et la valeur de la tâche. Avec des étudiants de niveau lycée, Pintrich (1989) a montré que les croyances de contrôle interne étaient positivement liées à l'utilisation de stratégies métacognitives et aux performances aux examens. Dans le cadre d'un entraînement visant le développement de stratégies de mémorisation et administré à 60 enfants de 6 à 8 ans, Kurtz et Borkowski (1987) ont observé que l'attribution des performances à des facteurs contrôlables (en particulier les efforts et les stratégies) montrait une relation positive avec l'utilisation subséquente de stratégies de mémorisation lors de tâches de transfert ainsi que lors de tâches de généralisation. Ces relations varient significativement en fonction de l'âge des élèves.

Les études de Sperling, Howard, Staley et alii (2004) auprès d'étudiants universitaires de première année ont montré que les scores obtenus à la mesure autorapportée de l'utilisation de stratégies métacognitives (Metacognitive Awareness Inventory ou MAI, voir Schraw \& Dennison, 1994) étaient positivement et significativement corrélés $(r=0,40 ; p<0,05)$ aux scores obtenus à des échelles de croyances motivationnelles (tirées du Motivated Strategy for Learning Questionnaire ou MSLQ, voir Pintrich, Smith, Garcia et al., 1993). Au contraire, la composante " connaissances métacognitives » du MAI n'était pas liée significativement aux échelles précitées. En particulier, la régulation de la cognition était corrélée plus fortement avec les buts de maîtrise, la perception de contrôle et le sentiment d'efficacité personnelle.

\section{Métacognition et perception des compétences}

La perception de ses compétences est essentielle à l'utilisation de stratégies métacognitives et était à l'origine considérée comme faisant partie des connaissances métacognitives sur soi (Pintrich, Wolters \& Baxter, 2000). Hacker et Bol (2004) postulent par exemple que le sentiment d'efficacité par rapport à la mémorisation influence les jugements de monitorage. Si une personne pense que sa capacité de mémorisation est faible, elle aura ainsi tendance à douter fortement de ses réponses dans une tâche de rappel. Au contraire, un fort sentiment d'efficacité pourrait aboutir à un optimisme ou une surestimation de ses performances. Par rapport aux stratégies métacognitives, un sentiment d'efficacité personnelle est nécessaire pour s'engager cognitivement dans une tâche et planifier ses actions. Selon Bandura (2003), les individus auraient besoin d'un sentiment d'efficacité pour appliquer leurs connaissances de façon cohérente et persévérante. Le manque de ce sentiment pourrait donc expliquer en partie le déficit de production de certains élèves. En effet, le guidage de l'activité de résolution nécessite des efforts cognitifs qu'un élève ne se percevant pas compétent aura tendance à ne pas produire. Pintrich (1999) rapporte, à propos d'une synthèse des recherches ayant utilisé le MSLQ (Pintrich, Smith, Garcia et al., 1993), des corrélations faibles à modérées entre le sentiment d'efficacité et l'utilisation de différents types de stratégies d'apprentissage (répétition, élaboration, organisation et stratégies métacognitives). L'amélioration du sentiment d'efficacité personnelle peut mener à une meilleure utilisation des stratégies cognitives et métacognitives (Bandura, 2003) et à un plus haut niveau 
d'accomplissement en mathématiques, en lecture et en écriture, comme l'indiquent les conclusions d'une synthèse de la littérature rédigée par Schunk (1989).

Dans nos travaux (Berger \& Karabenick, 2011), nous avons analysé de manière longitudinale l'influence du sentiment d'efficacité en mathématiques sur l'utilisation de stratégies d'apprentissage dans cette même matière, au cours d'un trimestre. À l'aide d'un modèle de type cross-lagged correlation (Kenny, 1979) et d'un échantillon de 306 élèves de 15 ans du niveau secondaire supérieur, nous avons observé que le sentiment d'efficacité prédit le changement dans la fréquence d'utilisation de stratégies métacognitives au cours du trimestre. En effet, le sentiment d'efficacité influence positivement l'utilisation de ces stratégies, même après avoir statistiquement contrôlé leur niveau d'utilisation au début du trimestre. Ainsi, un sentiment d'efficacité élevé est nécessaire tant à l'utilisation qu'au développement des stratégies métacognitives. Selon Bandura (2003), les élèves dont le sentiment d'efficacité est élevé auraient plus de facilité à développer des stratégies et les utiliseraient de façon plus efficace une fois acquises. II existe donc un effet de cette variable du domaine de la motivation sur le développement et l'utilisation des stratégies métacognitives. Bouffard-Bouchard (1990 ; Bouffard-Bouchard, Parent \& Larivée, 1990) a démontré expérimentalement que le sentiment d'efficacité personnelle est lié au degré d'exactitude des jugements de confiance en ses réponses, ceci indépendamment des capacités cognitives de l'individu. Ainsi, plus le sentiment d'efficacité personnelle est élevé, plus les jugements de confiance sont précis et réalistes.

\section{Métacognition et valeur de la tâche}

Pintrich (1999) rapporte des corrélations de nulles à modérées entre la valeur de la tâche (composée de perceptions relatives à l'importance, à l'utilité et à l'intérêt pour le cours) et les différentes stratégies d'apprentissage. Plusieurs recherches empiriques de Miller et ses collaborateurs (Brickman \& Miller, 1998, 2001 ; Miller, Greene, Montalvo et al., 1996), utilisant des études de cas et des échantillons de plusieurs centaines d'adolescents, ont montré que le niveau d'instrumentalité perçue était positivement lié aux comportements d'apprentissage autorégulé. En effet, l'instrumentalité perçue d'un cours pour l'atteinte de buts futurs est significativement prédictive des stratégies d'autorégulation et d'un mode de traitement profond des tâches, même après avoir contrôlé l'influence des buts de compétence et du sentiment d'efficacité personnelle. Lens, Simons et Dewitte (2002) ont quant à eux observé, dans un échantillon de 293 étudiants en première année de formation à l'enseignement, un effet du type d'instrumentalité perçu sur l'utilisation de stratégies d'apprentissage. Les étudiants qui s'engagent dans un cours pour des raisons qu'ils estiment pertinentes pour eux-mêmes (par exemple leur développement personnel) disent utiliser moins de stratégies dites de surface et plus de stratégies dites profondes (dont les stratégies métacognitives) que les élèves qui s'engagent dans un cours pour des raisons telles que l'obtention d'une récompense de la part de leurs parents.

D'autres travaux ont montré que le niveau d'intérêt tant individuel que situationnel est lié au degré d'exactitude des jugements métacognitifs (Tobias, 1994, 1995). Dans une recherche portant sur 139 étudiants universitaires (Tobias, 1995), les participants ont évalué dans quelle mesure ils avaient connaissance d'un certain nombre de termes médicaux tirés d'un texte scientifique (il s'agit d'un test nommé Knowledge Monitoring Assessment ${ }^{12}$ ou KMA). Ils ont, par la suite, été testés quant à leurs connaissances effectives de ces termes et leur intérêt pour le domaine. Les résultats ont révélé que l'intérêt individuel avait un effet faible mais significatif sur l'adéquation des jugements de confiance relatifs à la solution (c'est-à-dire l'éva- 
luation de sa réponse), même en contrôlant l'effet des connaissances antérieures. Par rapport à l'intérêt situationnel, une autre recherche de Tobias (1994) conclut que la personnalisation de problèmes de mathématiques (c'est-à-dire l'inclusion des noms des élèves ou de l'enseignant dans les données des problèmes) améliore l'adéquation des jugements métacognitifs, mais uniquement pour les élèves qui ont de faibles compétences en mathématiques. Le mécanisme ou les processus médiateurs entre l'intérêt et le monitorage restent inexpliqués, bien qu'une augmentation de l'attention et une plus grande persistance soient postulées comme facteurs explicatifs (Tobias, 1994 ; Tobias \& Everson, 2000).

\section{Métacognition et buts de compétence}

D'une manière générale, les différents buts de compétence sont liés à la qualité de l'engagement dans l'apprentissage. Un engagement actif se caractérise selon Ames (1992) par l'application de stratégies efficaces et l'utilisation de ces stratégies dépend notamment de la croyance que les efforts mènent au succès et que les échecs peuvent être « remédiés » par un changement dans les stratégies (perception de contrôle, voir Borkowski, Chan \& Muthukrishna, 2000).

Par rapport à la métacognition, de nombreuses études corrélationnelles (par exemple Ames \& Archer, 1988 ; Dweck \& Leggett, 1988 ; Galand \& Philippot, 2002 ; Schraw, Horn, Thorndike-Christ et al., 1995) ont montré que plus les élèves adoptent des buts de maîtrise, plus ils rapportent une utilisation fréquente de stratégies métacognitives telles que vérifier leur compréhension. Pintrich et Garcia (1991) ont montré avec le MSLQ que les étudiants universitaires (au nombre de 263 dans les domaines des sciences sociales et de la biologie) qui adoptent les cours avec des buts de maîtrise disent planifier plus largement la préparation de leurs études, guider plus fréquemment leur compréhension (soit réaliser plus fréquemment du monitorage) ou encore gérer leur temps et leur environnement d'apprentissage de manière plus stricte que les étudiants adoptant des buts de performance. Ce dernier type de but est, selon la synthèse de Pintrich (1999), négativement corrélé à l'utilisation de stratégies métacognitives. Afin d'étudier les relations entre buts de compétence et stratégies d'apprentissage en mathématiques et en langue, Dowson (2000) a établi un modèle d'équations structurelles et l'a testé avec un échantillon de 602 élèves australiens âgés de 12 à 15 ans. Ses résultats indiquent que l'adoption de buts de maîtrise est positivement liée à l'utilisation de stratégies cognitives et métacognitives, alors que l'adoption de buts de performance et d'approche est négativement liée aux stratégies métacognitives, et ceci au travers des deux domaines scolaires.

Dans deux études impliquant des étudiants de bachelor, Elliot, McGregor et Gable (1999) ont démontré que les différents buts de compétence sont liés de façon cohérente aux stratégies d'apprentissage. Les buts de maîtrise prédisent l'utilisation de stratégies impliquant un traitement profond (notamment des stratégies métacognitives) ; les buts de performance-approche prédisent un traitement en surface et les buts de performance-évitement prédisent un traitement profond moins fréquent et un traitement en surface plus fréquent, ainsi que la tendance à être désorganisé dans son travail.

Dermitzaki et Efklides (2003) ont quant à elles lié les buts de compétence définis par Nicholls (1989), soit l'orientation vers la tâche (analogue aux buts de maîtrise) et l'orientation vers l'ego (analogue aux buts de performance), avec le concept de soi, les expériences métacognitives (sentiment de difficulté, estimation de l'exactitude de la réponse et estimation des efforts) et les stratégies rapportées sur une tâche de résolution de problèmes mathématiques. Les participants de cette 
étude sont 512 élèves âgés de 12 à 16 ans. Les résultats montrent que les effets des aptitudes cognitives (compétences en raisonnement quantitatif), des performances et du concept de soi sur les expériences métacognitives sont largement plus forts que ne le sont les effets des buts de compétence. L'orientation vers la tâche a toutefois un effet direct sur les stratégies rapportées, puisque les élèves les plus orientés vers la tâche rapportent avoir utilisé plus de stratégies au cours du problème. Les chercheurs concluent que les buts de compétence sont liés à la performance scolaire plutôt qu'aux capacités cognitives. De plus, les buts de compétence exercent leurs effets sur les expériences métacognitives par l'intermédiaire du sentiment d'efficacité personnelle. Nos propres travaux (Berger, 2009) sur les relations entre buts de compétence et métacognition ont révélé, au contraire de l'étude de Dermitzaki et Efklides (2003), que les buts de compétence avaient des effets significatifs sur les expériences métacognitives et l'utilisation de stratégies métacognitives. Nos résultats, issus d'une étude à laquelle ont participé 253 élèves d'une moyenne d'âge de 18 ans, indiquent que les différents buts de compétence ont des effets spécifiques sur les expériences métacognitives, tant avant de débuter un problème mathématique qu'après avoir essayé de le résoudre. Par exemple, les buts de maîtrise prédisent les expériences métacognitives relatives aux efforts, l'estimation des efforts nécessaires pour résoudre le problème. Le recours à des stratégies métacognitives est indirectement prédit par les buts de compétence, l'effet de ces derniers étant médiatisé par les expériences métacognitives. Ces résultats montrent la grande complexité des effets des buts de compétence sur la métacognition.

Une étude de Nolen (1988) sur le même sujet a illustré, comme celles de Pintrich et ses collaborateurs, que les croyances motivationnelles entretenaient une relation indirecte avec la performance scolaire, ceci au travers de leurs liens avec l'utilisation de stratégies cognitives et métacognitives, c'est-à-dire un engagement cognitif considéré comme plus poussé. Cette étude, dont les 62 participants étaient âgés d'une quinzaine d'années, a montré que les buts de maîtrise, tant au niveau général (habitudes d'apprentissage) qu'au niveau d'un exercice spécifique, étaient associés à des stratégies dites de traitement profond alors que les buts de performance n'y étaient pas associés. En outre, les buts d'évitement du travail étaient négativement corrélés tant à l'utilisation de stratégies dites de traitement profond qu'à l'utilisation de stratégies dites de traitement en surface. Ceci signifie un engagement cognitif moindre.

Pintrich et ses collaborateurs (Pintrich, 1999 ; Wolters \& Pintrich, 1998 ; Wolters, Pintrich \& Karabenick, 2005 ; Wolters, Yu \& Pintrich, 1996) ont étudié à de multiples reprises les relations entre les buts de compétence et l'utilisation de divers types de stratégies d'apprentissage chez des adolescents et étudiants universitaires. D'une manière générale, leurs résultats indiquent que les élèves qui adoptent des buts de maîtrise rapportent une utilisation plus fréquente de stratégies de traitement profond (comme l'élaboration) et de stratégies métacognitives (planification, guidage de la compréhension, régulation, etc.) que les élèves adoptant des buts orientés vers la performance. En dépit de leurs effets sur le type de stratégies d'apprentissage mobilisées, les buts de maîtrise ne sont pas systématiquement bénéfiques pour les performances scolaires (Efklides \& Dina, 2007 ; Senko \& Miles, 2008). Par exemple, l'étude de Senko et Miles (2008) arrive à la conclusion que les 260 étudiants testés adoptant des buts de maîtrise tendent à allouer d'une manière disproportionnée leurs efforts aux cours qui les intéressent personnellement, ce qui produit un effet négatif sur l'ensemble de leurs notes car ils n'étudient pas suffisamment les autres cours. Au contraire, les étudiants adoptant des buts de performance ne présenteraient pas cette tendance. 


\section{Métacognition et anxiété}

Les recherches liant l'affect à la métacognition sont relativement rares. Toutefois, les travaux de Tobias et Everson, ceux de Veenman, ainsi que ceux de Dina et Efklides (2009) nous permettent d'éclairer un tant soit peu les relations entre anxiété, comme variable représentant généralement l'affect, et aspects métacognitifs. Tobias et Everson (2000) rapportent des corrélations significatives entre le KMA, l'échelle d'anxiété en mathématiques de Fennema et Sherman (1976) et la Worry-Emotionality Scale (Morris, Davis \& Hutchings, 1981). Par deux études transversales auprès de respectivement 117 étudiants de bachelor et 51 élèves de 10 ans aux États-Unis, ces chercheurs montrent que plus l'élève est précis dans la prédiction de sa propre réussite à un problème mathématique, moins il est anxieux ou angoissé par rapport aux mathématiques. Au contraire, les élèves qui sont le moins précis en monitorage sont plus fragiles émotionnellement dans les tâches de mathématiques. Les auteurs expliquent ces résultats en postulant que la représentation de l'anxiété occupe une partie des capacités cognitives, réduisant ainsi les capacités disponibles pour le raisonnement relatif au problème. En outre, ce phénomène est particulièrement prononcé chez les élèves ayant fortement tendance à être anxieux dans les situations de tests. Ainsi, une relation négative entre anxiété et exactitude des jugements métacognitifs n'est pas surprenante (Tobias \& Everson, 2000).

Si l'aspect matériel demande une forte capacité cognitive, l'anxiété et le monitorage semblent interagir pour influencer la performance. En outre, les élèves les plus anxieux, indépendamment de leurs compétences métacognitives, réalisent de moins bonnes performances dans des tâches de lecture qui demandent un fort investissement cognitif, ce qui suggère que les angoisses pourraient interférer avec l'utilisation de jugements et de stratégies métacognitives (Lafortune \& Pons, 2004) : l'anxiété et l'angoisse consomment une partie des capacités cognitives. Selon deux études conduites par Veenman, Kerseboom et Imthorn (2000) auprès de respectivement 30 et 75 adolescents de 12 et 13 ans aux Pays-Bas, les compétences métacognitives joueraient un rôle médiateur dans la relation entre l'anxiété en situation de test et la performance. Pour une partie des élèves, leur état d'anxiété amène, par des pensées interférant avec le fonctionnement cognitif, un déficit de production lors de la résolution de tâches mathématiques. De ce fait, leur performance dans ces tâches est réduite. Ainsi, l'état d'anxiété influence indirectement la performance via les compétences métacognitives. Pour un autre type d'élèves, c'est le manque de compétences métacognitives lui-même qui a pour effet de détériorer la performance et de créer chez eux un état d'anxiété. Dans ce cas, ce sont les compétences métacognitives qui ont une influence indirecte sur l'état d'anxiété via la performance. La relation entre les compétences métacognitives et l'anxiété serait ainsi fonctionnellement différente suivant les élèves.

Dina et Efklides (2009) se sont intéressées aux relations entre les expériences métacognitives et l'état d'anxiété lors de la résolution de problèmes mathématiques chez 870 adolescents de 12 à 15 ans fréquentant l'école obligatoire en Grèce. Plusieurs expériences métacognitives (le sentiment de difficulté, l'estimation des efforts nécessaires pour la résolution et le sentiment de confiance) contribuent, selon leurs résultats, à l'état d'anxiété ressenti par l'élève durant la résolution, ceci même après avoir statistiquement contrôlé les effets de toute une série de variables (le sexe, le sentiment d'efficacité, l'anxiété comme trait de personnalité ou encore les buts de compétence). Le sentiment de difficulté et l'estimation des efforts sont positivement associés à l'état d'anxiété car ils suggèrent un échec probable ainsi qu'un manque d'aisance dans le traitement de la tâche. Au contraire, le sentiment de confiance est négativement associé à l'état d'anxiété car il est un indicateur de l'adéquation de la réponse trouvée par l'élève. L'intérêt de ces résultats est qu'ils 
illustrent la complexité des interactions entre métacognition et affect en situation d'apprentissage, un champ de recherche qui reste encore largement inexploré. Les implications pratiques de telles recherches sont nombreuses; nous nous essayons à en formuler quelques-unes ci-après.

\section{CONCLUSION : IMPLICATIONS POUR L'ENSEIGNEMENT, L'APPRENTISSAGE ET LE DÉVELOPPEMENT DE LA MÉTACOGNITION}

Cette note de synthèse suggère qu'enseigner des compétences et des connaissances (par exemple des stratégies d'apprentissage ou de résolution de problème) sans se soucier des aspects motivationnels est peu prolifique. En effet, notre revue de la littérature souligne que les élèves doivent développer des croyances motivationnelles favorables, sans quoi leur engagement cognitif ne sera pas optimal et leur potentiel d'apprentissage ne s'exprimera pas pleinement. Ainsi, les élèves ne développeront pas leurs compétences et connaissances autant qu'ils en sont capables. Indubitablement, des croyances motivationnelles défavorables sont liées à un moindre exercice de stratégies métacognitives ainsi qu'à des jugements métacognitifs moins adaptés. Or ces deux aspects de la métacognition sont essentiels pour un apprentissage de qualité. Ceci implique, selon nous, que le développement de croyances motivationnelles favorables devrait faire partie intégrante des méthodes d'enseignement. Par exemple, le modèle de l'enseignement explicite (Gauthier, Bissonnette \& Richard, 2007) pourrait être étendu afin de favoriser non seulement la prise de conscience et l'objectivation des savoirs, mais aussi les croyances motivationnelles soutenant le maintien et le transfert des apprentissages.

Dans l'étude de la métacognition, de ses relations avec d'autres variables pertinentes pour l'apprentissage ou encore de son développement, l'accent a largement été mis sur deux des aspects de la métacognition, à savoir les connaissances métacognitives et les stratégies métacognitives, alors que les expériences métacognitives ont été passablement négligées par les chercheurs en psychologie de l'éducation. Toutefois les recherches, en particulier celles conduites par Efklides, ont récemment montré que cet aspect de la métacognition constitue un facteur explicatif majeur dans l'engagement cognitif des élèves (notamment dans la mobilisation de stratégies métacognitives) ainsi que dans l'état d'anxiété. En effet, les expériences métacognitives jouent un rôle médiateur entre croyances motivationnelles et engagement cognitif. Elles constituent ainsi un aspect auquel les enseignants devraient porter attention et qui devrait être stimulé en classe. Ceci peut se réaliser simplement en demandant aux élèves de réaliser des jugements et d'exprimer leurs sentiments avant de débuter une tâche ou après avoir proposé une solution à cette tâche. Les élèves prenant l'habitude de juger différents aspects relatifs à leur fonctionnement cognitif lors des tâches scolaires ont ensuite la possibilité de se servir de ces indices pour utiliser des stratégies métacognitives de manière plus optimale et développer leurs connaissances métacognitives. Si l'on cherche à développer les aspects métacognitifs (par exemple l'utilisation de stratégies métacognitives), il est nécessaire de s'attaquer aux obstacles motivationnels également, sans quoi les efforts peuvent s'avérer vains. Ainsi les interventions de type éducation cognitive (Büchel, 1995) ne peuvent pas se limiter à cibler les aspects cognitifs et métacognitifs de l'apprentissage. Ces interventions devraient également avoir l'ambition de développer des croyances motivationnelles facilitant l'apprentissage, l'utilisation et le transfert des stratégies d'apprentissage cognitives et métacognitives. 
Plusieurs études réalisées à l'université de Genève ont eu pour objectif de développer les stratégies d'apprentissage et les aspects métacognitifs chez des enfants et adolescents rencontrant des difficultés d'apprentissage conséquentes (pour des études récentes, voir par exemple Berger, Kipfer \& Büchel, 2008, 2010 ; Bosson, Hessels, Hessels-Schlatter et al., 2010 ; Büchel, Berger, Kipfer et al., 2010 ; Roh, 2012). Les interventions réalisées auprès d'élèves âgés de 8 à 18 ans ont toutes suivi des principes identiques et étaient axées sur des aspects cognitifs principalement (pour une description des fondements et des principes des interventions, voir Büchel, 1995, 1999, 2007). Étant donné que les interventions de type éducation cognitive permettent d'améliorer les processus d'apprentissage, nous pourrions attendre de ces entraînements qu'ils aient un effet de facto sur certaines croyances motivationnelles. Bien que les intervenants (chercheurs ou enseignants en fonction des études) aient été passablement informés au sujet des croyances motivationnelles et de leur importance, ceci ne constituait toujours qu'un aspect secondaire des interventions. En outre, les méthodes pour l'amélioration des croyances motivationnelles n'étaient pas prédéfinies et ainsi non appliquées systématiquement. Le risque est de ce fait que les effets de l'intervention ne soient pas aussi forts et généralisés qu'escompté. La maintenance et le transfert des apprentissages pourraient ainsi être limités si les élèves ne modifient pas suffisamment leurs croyances motivationnelles. Les croyances motivationnelles, évaluées par questionnaire avant et après l'intervention, n'ont ainsi pas été modifiées de manière statistiquement significative par ces interventions ${ }^{13}$. Ces résultats ne sont pas inhabituels ${ }^{14}$ (Paris \& Oka, 1986) : Zimmerman et Schunk (2004) relèvent que si les recherches ont montré que les entraînements de type éducation cognitive avaient un effet sur les processus d'apprentissage, elles ne permettent pas systématiquement d'améliorer les croyances motivationnelles, ce qui limite l'impact de ce type d'intervention.

À l'inverse, un entraînement pour améliorer les aspects motivationnels qui n'est pas accompagné d'un entraînement stratégique n'améliorerait pas les processus d'apprentissage et n'aurait par conséquent que peu d'influence sur les performances scolaires. Il semble donc important de travailler sur les deux domaines dans le cadre des entraînements de type stratégique. C'est pourquoi les interventions relevant de l'éducation cognitive devraient s'efforcer d'associer l'entraînement stratégique (enseignement de stratégies d'apprentissage et développement des aspects métacognitifs) avec une intervention au niveau motivationnel (par exemple la modification du style attributif ou le renforcement du sentiment d'efficacité personnelle). Les croyances motivationnelles qui font barrière à un engagement stratégique de l'élève, telles qu'un style attributif de type incontrôlable ou un faible sentiment d'efficacité, doivent être modifiées, et ceci passe non seulement par le fait de vivre des réussites scolaires mais aussi par la compréhension des raisons qui mènent à ces réussites. Les bons résultats doivent être attribués à des facteurs contrôlables (efforts et stratégies), tout comme les mauvais résultats. À ce propos, soulignons que le modèle de Borkowski (Borkowski, Chan \& Muthukrishna, 2000) met l'accent sur le complément ou l'articulation nécessaire des compétences ou connaissances tant métacognitives que motivationnelles pour améliorer les résultats de ces entraînements. Relevons que, si l'articulation entre croyances motivationnelles et composantes de la métacognition est décrite comme une causalité réciproque (influences bidirectionnelles) dans les modèles théoriques de Borkowski et de Pintrich, les recherches ont généralement conceptualisé la relation comme unidirectionnelle. Les travaux ont en effet le plus souvent étudié l'influence des croyances motivationnelles sur la métacognition, négligeant l'influence opposée. Ceci est sans doute dû au fait que les plans de recherche généralement adoptés sont de type transversal, et au fait que les stratégies métacognitives (et plus généralement l'autorégulation de l'apprentissage) constitueraient un médiateur de la relation entre croyances motivationnelles 
et performance. Des études longitudinales, dans lesquelles les croyances motivationnelles et les aspects métacognitifs seraient mesurés à plusieurs reprises sur une certaine période, sont nécessaires afin d'appréhender la causalité réciproque telle qu'évoquée dans les modèles théoriques. De plus, il est probable que certaines croyances motivationnelles soient plus difficilement modifiables que d'autres.

Nous avons constaté, dans les recherches mentionnées ainsi que dans des séances de supervision d'enseignants, d'éducateurs et de maîtres d'apprentissage engagés dans des centres de formation professionnelle destinés aux apprentis de faibles compétences scolaires, que les apprenants n'étaient que rarement invités à réfléchir sur leurs motivations et leurs sentiments avant de commencer un travail et aussi pendant son exécution. Pour cette raison, nous avons introduit, dans la nouvelle édition du programme d'apprentissage DELV (Büchel \& Büchel, 2011), de manière explicite et répétée, des questions concernant diverses croyances motivationnelles (effets attendus de la tâche, attributions causales, sentiment d'efficacité personnelle) et les expériences métacognitives associées.

En conclusion, mentionnons quelques pistes à suivre pour favoriser le développement non seulement de la métacognition, mais aussi du sentiment d'efficacité personnelle. Les travaux de Schunk ont combiné l'enseignement et la verbalisation de stratégies avec des feedbacks attribuant les performances aux efforts (Schunk, 1982). Cette procédure s'est révélée bénéfique au développement du sentiment d'efficacité personnelle chez des élèves rencontrant des difficultés d'apprentissage. En outre, apprendre à l'élève à se fixer des buts pouvant être atteints à court terme (par exemple réaliser un certain nombre d'exercices de division dans la demi-heure à venir) plutôt que des buts atteignables à long terme (par exemple obtenir une bonne note à l'épreuve ayant lieu dans un mois) est une autre manière d'augmenter le sentiment d'efficacité puisque la réussite de multiples buts proximaux atteste d'un accroissement de ses compétences (Schunk \& Pajares, 2005 ; Locke \& Latham, 2002). Finalement, le modelage, consistant pour l'enseignant à servir de modèle afin d'aider les élèves dans l'acquisition de certaines compétences, permet non seulement d'expliciter les processus de pensée, développant ainsi la réflexion sur le fonctionnement cognitif, mais aussi d'améliorer le sentiment d'efficacité personnelle. Ceci est en particulier le cas si l'élève peut s'identifier au modèle car celui-ci fait des fautes et exprime des doutes sur son raisonnement (Schunk \& Ertmer, 2000). Ainsi les enseignants pourraient recourir au modelage réalisé par des pairs afin de favoriser le sentiment d'efficacité des élèves les plus faibles.

$$
\begin{array}{r}
\text { Jean-Louis Berger } \\
\text { Jean-Louis.Berger@iffp-suisse.ch } \\
\text { Institut fédéral des hautes études en formation professionnelle (Suisse) }
\end{array}
$$

Fredi Büchel Fredi.Buchel@unige.ch Université de Genève (Suisse), Faculté de psychologie et des sciences de l'éducation 


\section{NOTES}

1 Ces modèles considèrent par exemple les stratégies métacognitives, les stratégies cognitives et/ou la mémoire de travail comme les facteurs prépondérants du fonctionnement intellectuel.

2 Cette perspective sur la cognition a été qualifiée de « cognition froide » (cold cognition) par opposition à une « cognition chaude », qui se réfère au fait que l'affect accompagne ou qualifie l'information (Zajonc, 1980).

3 Dans un souci de simplification du texte, seule la forme masculine est employée dans certains passages du texte. Il est toutefois évident que les deux genres sont considérés de manière totalement égalitaire.

4 Ce que nous pouvons traduire par : « un enchaînement d'actions en vue d'atteindre un but ou d'accomplir une tâche ".

5 Ce que nous pouvons traduire par : « la cognition scolaire est froide en ce qu'elle porte principalement sur les connaissances et stratégies nécessaires à l'efficience, alors que l'accent est peu placé sur les facteurs émotionnels qui pourraient promouvoir ou restreindre cette efficience ».

6 Ce que nous pouvons traduire par: « étant donné que la métacognition nécessite une capacité introspective au sujet de ses propres performances et [...] que l'auto-évaluation de sa propre performance ne peut pas être objective - un tel auto-questionnement est contaminé par son propre sentiment de compétence -, des domaines du développement de la personnalité qui étaient auparavant distincts sont à nouveau d'une pertinence évidente (comme la peur de l'échec, le besoin d'accomplissement, le contrôle interne vs externe, l'incapacité apprise et le niveau d'aspiration) ".

7 L'agentivité se réfère à la perception de nos propres actes comme étant effectivement causés et contrôlés par nous-mêmes.

8 Ce que nous pouvons traduire par : « II est clair que ses propres croyances sur la mémoire et les variables qui l'affectent devraient contribuer aux jugements métacognitifs dans toute situation ».

9 Selon Fenigstein, Scheier et Buss (1975), concevoir l'implication du soi dans le comportement sans considérer qu'il existe deux facettes du soi serait simpliste. Ils proposent de distinguer deux types de soi : le soi privé, qui consiste en ce que nous sommes et ce que nous ressentons intérieurement et le soi public, qui est l'identité que nous présentons aux autres. Les jugements métacognitifs peuvent ainsi différer suivant leur réalisation privée ou publique.

10 Les modèles d'autorégulation récents sont très fortement influencés par le constructivisme, ce qui rejoint le postulat d'une orientation constructiviste pour toute intervention métacognitive (Pressley, Harris \& Marks, 1992). Néanmoins, nous ne devrions pas oublier que les axiomes principaux des modèles d'autorégulation se trouvaient déjà dans des programmes d'apprentissage relativement anciens. À titre d'illustration, Dansereau et ses collaborateurs (voir par exemple Dansereau, 1978 ; Dansereau, McDonald, Collins et al., 1979) distinguent les stratégies primaires des stratégies de soutien. Ces dernières se composent de facteurs qui devraient créer et maintenir un climat favorable à l'apprentissage ; la plupart sont des facteurs que nous appelons aujourd'hui des croyances motivationnelles, par exemple l'estime de soi (proche du concept de sentiment d'efficacité). Toutefois, ce modèle a été critiqué pour son efficacité insuffisante (Büchel, 1983). Une conjonction de variables de la motivation et de variables cognitives a également été proposée par Keller, Binder et Thiel (1981) sous forme de programme d'entraînement. Les bases théoriques de ce programme sont toutefois assez éclectiques et l'absence d'une théorie intégrative ne peut pas être ignorée.

11 Ce que nous pouvons traduire par : « traitement subconscient, autonome, avec des intentions, habitudes passées, pensées et souvenirs ».

12 Dans ce test, l'élève doit prédire sa réussite à des exercices qu'il va devoir ensuite réaliser. Ces prédictions sont ensuite comparées à la performance effectivement réalisée par l'élève. Les exercices sont tout d'abord montrés à l'élève afin qu'il puisse réaliser les prédictions (jugements métacognitifs). La différence entre la performance de l'élève et ses prédictions informe sur sa capacité à réaliser des jugements métacognitifs exacts ainsi que sur la précision de ces jugements.

13 Ces résultats sont partiellement dus au fait que les informations sont auto-rapportées par les élèves. De ce fait, plusieurs biais peuvent se produire, ajoutant une part de variance d'erreur dans la mesure des croyances motivationnelles. Les élèves peuvent surestimer ou sous-estimer leurs propres croyances motivationnelles afin de conserver une image de soi favorable, que ce soit auprès d'eux-mêmes ou vis-àvis des autres. De plus, la désirabilité sociale de certaines croyances motivationnelles telles que l'orientation des buts vers la maîtrise ou l'interprétation des items de façon différente de celle attendue par le chercheur (Karabenick, Wooley, Friedel et al., 2007) sont autant d'autres facteurs pouvant biaiser la mesure des croyances motivationnelles lorsque celles-ci sont auto-rapportées.

14 Dans le cadre d'une étude évaluant les effets d'un programme d'intervention visant à améliorer les connaissances métacognitives ainsi que les stratégies pour la compréhension de texte chez environ un millier d'élèves de 8 et 10 ans, Paris et Oka (1986) ont postulé que le programme aurait également des effets sur la façon dont les élèves perçoivent leurs compétences. En effet, si les élèves acquièrent des compétences stratégiques, ils devraient par conséquent se percevoir comme plus compétents en compréhension de texte. Bien que des effets significatifs de ce programme aient été observés sur les connaissances métacognitives et les stratégies, aucun effet sur la perception des compétences n'a pu être mis en exergue, ceci malgré la longueur du programme (jusqu'à quarante heures). 


\section{BIBLIOGRAPHIE}

AMES C. (1992). «Classrooms : Goals, structures, and student motivation ». Journal of Educational Psychology, vol. 84, no 3, p. 261-271.

AMES C. \& ARCHER J. (1988). «Achievement goals in the classroom: Students learning strategies and motivation processes ». Journal of Educational Psychology, vol. 80, n० 3, p. 260-267.

BADDELEY A. \& LOGIE R. (1999). «Working memory: The multiple-component model ». In A. Miyake \& P. Shah (dir.), Models of working memory. Cambridge : Cambridge University Press, p. 28-61.

BANDURA A. (1986). Social foundations of thought and action: A social cognitive theory. Englewood Cliffs : Prentice Hall.

BANDURA A. (2003). Auto-efficacité. Le sentiment d'efficacité personnelle. Bruxelles : De Boeck.

BEREITER C. \& SCARDAMALIA M. (1985). "Cognitive coping strategies and the problem of "inert knowledge" ". In S. Chipman, J. Segal \& R. Glaser (dir.), Thinking and learning skills: Research and open questions, vol. 2. Hillsdale : Erlbaum, p. 65-80.

BERGER J.-L. (2008). Motivation, métacognition et aptitudes cognitives chez les apprenants de la formation professionnelle initiale. Thèse de doctorat, sciences de l'éducation, Université de Genève, Suisse.

BERGER J.-L. (2009). "The influence of achievement goals on metacognitive processes in math problem solving ". Journal of Cognitive Education and Psychology, vol. 8, n० 2, p. 165-181.

BERGER J.-L. \& KARABENICK S. (2011). “ Motivation and students' use of learning strategies: Evidence of unidirectional influences in mathematics classrooms ». Learning and Instruction, vol. 21, no 4, p. 416-428.

BERGER J.-L., KIPFER N. \& BÜCHEL F. (2008). "The effects of a metacognitive intervention for students in low performing vocational training tracks ». Journal of Cognitive Education and Psychology, vol. 8, no 1, p. 337-367.

BERGER J.-L., KIPFER N. \& BÜCHEL F. (2010). « Un modèle d'intervention métacognitive pour les apprenants en formation professionnelle initiale de deux ans. Principes d'application collective et efficacité ". In M. Hessels \& C. Hessels-Schlatter (dir.), Évaluation et intervention auprès des élèves en difficultés. Berne : Peter Lang, p. 159174.

BOBROW D. \& NORMAN D. (1975). «Some principles of memory schemata ». In D. Bobrow \& A. Collins (dir.), Representation and understanding: Studies in cognitive science. New York : Academic Press, p. 131-149.

BOEKAERTS M. (1996). " Self-regulated learning at the junction of cognition and motivation ». European Psychologist, vol. 1, n० 2, p. 100-112.

BOEKAERTS M. (1997). «Self-regulated learning: A new concept embraced by researchers, policy makers, educators, teachers, and students ». Learning and Instruction, vol. 7, n० 2, p. 161-186.

BOEKAERTS M. (1999). "Metacognitive experiences and motivational state as aspects of self-awareness: Review and discussion ". European Journal of Psychology of Education, vol. 14, no 4, p. 571-584.

BOEKAERTS M. (2010). "Motivation et émotion : deux piliers de l'apprentissage en classe ». In H. Dumont, D. Istance \& F. Benavides (dir.), Comment apprend-on ? La recherche au service de la pratique. Paris: OCDE, p. 97-119.

BORKOWSKI J., CHAN L. \& MUTHUKRISHNA N. (2000). «A process-oriented model of metacognition: Links between motivation and executive functioning ". In G. Schraw \& J. Impara (dir.), Issues in the measurement of metacognition. Lincoln : Buros Institute, p. 1-41.

BORKOWSKI J., JOHNSTON M. \& REID M. (1987). « Metacognition, motivation, and controlled performance ". In S. Ceci (dir.), Handbook of cognitive, social, and neuropsychological aspects of learning disabilities, vol. 2. Hillsdale : Erlbaum, p. 147173.

BORKOWSKI J., MILSTEAD M. \& HALE C. (1988). « Components of children's metamemory: Implications for strategy generalization ». In F. Weinert \& M. Perlmutter (dir.), Memory development: Universal changes and individual differences. Hillsdale : Erlbaum, p. 72100. 
BORKOWSKI J., REID M. \& KURTZ B. (1984). « Metacognition and retardation: Paradigmatic, theoretical, and applied perspectives ". In P. Brooks, R. Sperber \& C. McCauley (dir.), Learning and cognition in the mentally retarded. Hillsdale : Erlbaum, p. 55-75.

BORKOWSKI J., WEYHING R. \& CARR M. (1988). «Effects of attributional retraining on strategy-based reading comprehension in learning-disabled students ». Journal of Educational Psychology, vol. 80, no 1, p. 46-53.

BOSSON M., HESSELS M., HESSELS-SCHLATTER C., BERGER J.-L., KIPFER N. \& BÜCHEL F. (2010). "Strategy acquisition by children with learning difficulties in a metacognitive training ». Australian Journal of Learning Difficulties, vol. 15, n० 1 , p. 1-22.

BOUFFARD-BOUCHARD T. (1990). «Influence of self-efficacy on performance in a cognitive task ». Journal of Social Psychology, vol. 130, n³, p. 353-363.

BOUFFARD-BOUCHARD T., PARENT S. \& LARIVÉE S. (1990). "Capacité cognitive, sentiment d'auto-efficacité et autorégulation ». European Journal of Psychology of Education, vol. 5, no 3, p. 355-364.

BRICKMAN S. \& MILLER R. (1998). "Valuing of future goals and instrumentality as predictors of cognitive engagement ". Communication présentée au $6^{\text {th }}$ workshop on achievement and task motivation, Thessalonique, Grèce.

BRICKMAN S. \& MILLER R. (2001). "The impact of sociocultural knowledge on future goals and self-regulation ". In D. Mclnerney \& S. Van Etten (dir.), Research on sociocultural influences on motivation and learning. Greenwich : Information Age Publishing, p. 119137.

BROWN A. (1978). "Knowing when, where, and how to remember: A problem of metacognition ". In R. Glaser (dir.), Advances in instructional psychology, vol. 1. Hillsdale : Erlbaum, p. 77-165.

BROWN A. (1987). "Metacognition, executive control, self-regulation, and other more mysterious mechanisms ». In F. Weinert \& R. Kluwe (dir.), Metacognition, motivation, and understanding. Hillsdale : Erlbaum, p. 65-116.

BROWN A., BRANSFORD J., FERRARA R. \& CAMPIONE J. (1983). « Learning, remembering, and understanding ". In J. Flavell \& E. Markman (dir.), Handbook of child psychology. Vol. 3: Cognitive development. New York: Wiley, p. 77-166.

BÜCHEL F. (1983). Lernstrategien bei Jugendlichen und Erwachsenen in der beruflichen Ausbildung. Thèse d'habilitation, psychologie générale, Université de Bâle, Suisse.

BÜCHEL F. (1991). " How are learning activities initiated? An epistemological view of mediated learning experience ". In R. Feuerstein, P. Klein \& A. Tannenbaum (dir.), Mediated learning experience: Theoretical, psychological and learning implications. Tel Aviv et Londres : Freund Publishing House, p. 53-70.

BÜCHEL F. (1995). “ De la métacognition à l'éducation cognitive ». In F. Büchel (dir.), L'éducation cognitive. Le développement de la capacité d'apprentissage et son évaluation. Neuchâtel : Delachaux \& Niestlé, p. 9-44.

BÜCHELF. (1996). «DELF : un programme métacognitif destiné à la formation professionnelle des adolescents et jeunes adultes ayant des difficultés d'apprentissage ». In A.M. Besse \& K. Bernath (dir.), Quelles chances sur le marché du travail ? A propos de la formation des formateurs de jeunes et adultes handicapés. Lucerne : SZH/SPC, p. 183-213.

BÜCHEL F. (1999). " DELF : un programme métacognitif pour adolescents en formation professionnelle ». In P.-A. Doudin, D. Martin \& O. Albanese (dir.), Métacognition et éducation. Aspects transversaux et disciplinaires. Berne : Peter Lang, p. 179-200.

BÜCHEL F. (2007). L'intervention cognitive en éducation spéciale : deux programmes métacognitifs. Genève : Université de Genève, coll. "Carnets des sciences de l'éducation ".

BÜCHEL F. \& BÜCHEL P. (2009). Das Eigene Lernen Verstehen. Handbuch zum DELVProgramm. Berne : HEP Verlag.

BÜCHEL F. \& BÜCHEL P. (2011). DELV : comprendre son propre apprentissage. Un programme pour améliorer l'apprentissage et le raisonnement des adolescents et des adultes. Tegna, Suisse : Centre d'éducation cognitive.

BÜCHEL F., BERGER J.-L., KIPFER N. \& FRAUCHIGER V. (2010). " Intervention métacognitive chez des adolescents avec retard mental modéré ". In $\mathrm{M}$. Hessels \& C. Hessels-Schlatter (dir.), Évaluation et intervention auprès des élèves en difficultés. Berne : Peter Lang, p. 129-144. 
CARR M., BORKOWSKI J. \& MAXWELL S. (1991). " Motivational components of underachievement ». Developmental Psychology, vol. 27, n० 1, p. 108-118.

COSNEFROY L. (2010). "Se mettre au travail et y rester : les tourments de l'autorégulation ". Revue française de pédagogie, no 170, p. 5-15.

COSNEFROY L. (2011). L'apprentissage autorégulé. Entre cognition et motivation. Grenoble : Presse universitaires de Grenoble.

COSNEFROY L. \& FENOUILLET F. (2009). "Motivation et apprentissages scolaires ». In P. Carré \& F. Fenouillet (dir.), Traité de psychologie de la motivation. Paris : Dunod, p. 127-145.

CRAIK F. \& LOCKHART R. (1972). " Levels of processing: A framework for memory research ». Journal of Verbal Learning and Verbal Behavior, vol. 11, n 6, p. 671-684.

CROMBACH M., BOEKAERTS M. \& VOETEN M. (2003). "Online measurements of appraisals of students faced with curricular tasks ". Educational and Psychological Measurement, vol. 63, no 1, p. 96-111.

DAI D. \& STERNBERG R. (2004). «Beyond cognitivism: Toward an integrated understanding of intellectual functioning and development ». In D. Dai \& R. Sternberg (dir.), Motivation, emotion, and cognition. Integrative perspectives on intellectual functioning and development. Mahwah : Erlbaum, p. 3-38.

DANSEREAU D. (1978). «The development of a learning strategies curriculum ». In H. O'Neil (dir.), Learning strategies. New York: Academic Press, p. 1-29.

DANSEREAU D., McDONALD B., COLLINS K., GARLAND J., HALLEY C., DIEKHOF G. \& EVANS S. (1979). "Evaluation of a learning strategy system ". In H. O'Neil \& C. Spielberger (dir.), Cognitive and affective learning strategies. New York : Academic Press, p. 3-43.

DAY J., BORKOWSKI J., DIETMEYER D., HOWSEPIAN B. \& SAENZ D. (1992). "Possible selves and academic achievement ". In L. Winegar \& J. Valsiner (dir.), Children's development within social context: Research and methodology, vol. 2. Hillsdale : Erlbaum, p. 181-201.

DE CORTE E., VERSCHAFFEL L. \& OP'T EYNDE P. (2000). «Self-regulation. A characteristic and a goal of mathematics education ». In M. Boekaerts, P. Pintrich \& M. Zeidner (dir.), Handbook of self-regulation. San Diego : Academic Press, p. 687-726.

DERMITZAKI I. \& EFKLIDES A. (2003). "Goal orientations and their effect on selfconcept and metacognition in adolescence ". Psychology: The Journal of the Hellenic Psychological Society, vol. 10, no 2-3, p. 214-227.

DINA F. \& EFKLIDES A. (2009). « Metacognitive experiences as the link between situational characteristics, motivation, and affect in self-regulated learning ". In M. Wosnitza, S. Karabenick, A. Efklides \& P. Nenniger (dir.), Contemporary motivation research: From global to local perspectives. Gottingen : Hogrefe \& Huber, p. 117-146.

DOWSON M. (2000). Relations between student's academic motivation, cognition, and achievement in Australian school settings. Thèse de doctorat non publiée, éducation, University of Western Sydney, Australie.

DWECK C. \& LEGGETT E. (1988). «A social-cognitive approach to motivational and personality ». Psychological Review, vol. 95, no 2, p. 256-273.

EFKLIDES A. (2001). "Metacognitive experience in problem solving ". In A. Efklides, J. Kuhl \& R. Sorrentino (dir.), Trends and prospects in motivation research. Dordrecht: Kluwer, p. 297-323.

EFKLIDES A. (2006). "Metacognition and affect: What can metacognitive experiences tell us about the learning process? » Educational Research Review, vol. 1, no 1, p. 3-14.

EFKLIDES A. (2008). "Metacognition: Defining its facets and levels of functioning in relation to self-regulation and co-regulation ". European Psychologist, vol. 13, n० 4, p. 277-287.

EFKLIDES A. \& DINA F. (2007). "Is mastery orientation always beneficial for learning? " In F. Salili \& R. Hoosain (dir.), Culture, motivation and learning: A multicultural perspective. Greenwich : Information Age Publishing, p. 131-167.

ELLIOT A., McGREGOR H. \& GABLE S. (1999). " Achievement goals, study strategies, and exam performance: A mediational analysis ". Journal of Educational Psychology, vol. 91, no 3, p. 549-563.

FENNEMA E. \& SHERMAN J. (1976). «Fennema-Sherman mathematics attitude scales ». Journal for Research in Mathematics Education, vol. 7, n० 5, p. 324-326. 
FENIGSTEIN A., SCHEIER M. \& BUSS A. (1975). "Public and private self-consciousness: Assessment and theory ". Journal of Consulting and Clinical Psychology, vol. 43, n० 4, p. 522-527.

FLAVELL J. (1976). «Metacognitive aspects of problem solving ». In L. Renick (dir.), The nature of intelligence. Hillsdale : Erlbaum, p. 231-235.

FLAVELL J. (1979). "Metacognition and cognitive monitoring. A new area of cognitivedevelopmental inquiry ». American Psychologist, vol. 34, no 10, p. 906-911.

FLAVELL J. (1987). "Speculations about the nature and development of metacognition ». In F. Weinert \& R. Kluwe (dir.), Metacognition, motivation, and understanding. Hillsdale : Erlbaum, p. 21-30.

GALAND B. \& BOURGEOIS É. (2006). (Se) motiver à apprendre. Paris : PUF.

GALAND B. \& PHILIPPOT P. (2002). "Style motivationnel des élèves du secondaire : développement d'un instrument de mesure et relations avec d'autres variables pédagogiques ". Revue canadienne des sciences du comportement, vol. 34, n० 4, p. 261-275.

GAUTHIER C., BISSONNETTE S. \& RICHARD M. (2007). « L'enseignement explicite ». In V. Dupriez \& G. Chapelle (dir.), Enseigner. Paris : PUF, p. 107-116.

HACKER D. \& BOL L. (2004). " Metacognitive theory: Considering the social-cognitive influences ». In D. Mclnerney \& S. Van Etten (dir.), Research on sociocultural influences on motivation and learning. Vol. 4: Big theories revisited. Greenwich : Information Age Publishing, p. 275-297.

KARABENICK S. \& BERGER J.-L. (2012). " Help seeking as a self-regulated learning strategy ». In H. Bembenutty, T. Cleary \& A. Kitsantas (dir.), Applications of selfregulated learning across diverse disciplines: A tribute to Barry J. Zimmerman. Greenwich : Information Age Publishing.

KARABENICK S., WOOLEY M., FRIEDEL J. et al. (2007). "Cognitive processing of selfreport items in educational research: Do they think what we mean? " Educational Psychologist, vol. 42, no 3, p. 139-151.

KELLER G., BINDER A. \& THIEL R. (1981). Lern- und Arbeitsverhaltenstraining LAZ für Schüler ab 14 Jahren. Braunschweig : Westermann.

KENNY D. (1979). Correlation and causality. New York : Wiley-Interscience.

KLUWE R. (1982). "Cognitive knowledge and executive control: Metacognition ". In D. Griffin (dir.), Animal mind. Human mind. New York: Springer-Verlag, p. 201-224.

KORIAT A. (2002). « Metacognition research: An interim report ». In T. Perfect \& B. Schwartz (dir.), Applied metacognition. Cambridge : University Press, p. 261-286.

KREUTZER M., LEONARD S. \& FLAVELL J. (1975). «An interview study of children's knowledge about memory ". Monographs of the Society for Research in Child Development, vol. 40, no 1, p. 1-57.

KURTZ B. \& BORKOWSKI J. (1987). " Development of strategic skills in impulsive and reflective children: A longitudinal study of metacognition ". Journal of Experimental Child Psychology, vol. 43, no 1, p. 129-148.

LAFORTUNE L. \& PONS F. (2004). "Le rôle de l'anxiété dans la métacognition. Une réflexion vers des actions ». In L. Lafortune, P.-A. Doudin, F. Pons \& D. Hancock (dir.), Les émotions à l'école. Sainte-Foy: Presses universitaires du Québec, p. 145-169.

LAVEAULT D., LEBLANC R. \& LEROUX J. (1999). “ Autorégulation de l'apprentissage scolaire : interaction entre processus métacognitifs et déterminants de la motivation ». In C. Depover \& B. Noël (dir.), L'évaluation des compétences et des processus cognitifs. Modèles, pratiques et contextes. Bruxelles: De Boeck, p. 81-98.

LENS W., SIMONS J. \& DEWITTE S. (2002). «From duty to desire: The role of students" future time perspective and instrumentality perceptions for study motivation and self-regulation ». In F. Pajares \& T. Urdan (dir.), Academic motivation of adolescents. Greenwich : Information Age Publishing, p. 221-245.

LOCKE E. \& LATHAM G. (2002). «Building a practically useful theory of goal setting and task motivation: A 35-year odyssey ». American Psychologist, vol. 57, no 9, p. 705-717.

MANDELMAN S., TAN M., KORNILOV S., STERNBERG R. \& GRIGORENKO E. (2010). « The metacognitive component of academic self-concept: The development of a triarchic self-scale ". Journal of Cognitive Education and Psychology, vol. 9, n० 1, p. 73-86.

MARKUS H. \& WURF E. (1987). "The dynamic self-concept: A social psychological perspective ». Annual Review of Psychology, vol. 38, p. 299-337. 
MARKUS H., CROSS S. \& WURF E. (1990). "The role of self-system in competence ". In R. Sternberg \& J. Kolligian Jr. (dir.), Competence considered. New Haven : Yale University Press, p. 205-225.

METALLIDOU P. \& EFKLIDES A. (2001). «The effects of general success-related beliefs and specific metacognitive experiences on causal attributions ». In A. Efklides, J. Kuhl, \& R. Sorrentino (dir.), Trends and prospects in motivation research. Dordrecht : Kluwer, p. 325-347.

MILLER R., GREENE B., MONTALVO G., RAVINDRAN B. \& NICHOLS J. (1996). « Engagement in academic work: The role of learning goals, future consequences, pleasing others, and perceived ability ". Contemporary Educational Psychology, vol. 21, n० 4, p. 388422.

MORRIS L., DAVIS M. \& HUTCHINGS C. (1981). "Cognitive and emotional components of anxiety: Literature review and a revised worry-emotionality scale ". Journal of Educational Psychology, vol. 73, no 4, p. 541-555.

MURPHY P. \& ALEXANDER P. (2000). «A motivated exploration of motivation terminology ». Contemporary Educational Psychology, vol. 25, no 1, p. 3-53.

NELSON T. (1996). "Consciousness and metacognition ». American Psychologist, vol. 51, n०2, p. 102-116.

NELSON T. \& NARENS L. (1990). " Metamemory: A theoretical framework and new findings ». In G. Bower (dir.), The psychology of learning and motivation, vol. 26. New York : Academic Press, p. 125-173.

NICHOLLS J. (1989). The competitive ethos and democratic education. Cambridge : Harvard University Press.

NOLEN S. (1988). «Reasons for studying: Motivational orientations and study strategies ». Cognition and Instruction, vol. 5, n० 4, p. 269-287.

NORMAN D. (1979). Slips of the mind and an outline for a theory of action. Report No. 88. San Diego : University of California, Center for Human Information Processing.

PARIS S. \& OKA E. (1986). «Children's reading strategies, metacognition, and motivation ». Developmental Review, vol. 6, no 1, p. 25-56.

PARIS S. \& WINOGRAD P. (1990). " How metacognition can promote academic learning and instruction ". In B. Jones \& L. Idol (dir.), Dimensions of thinking and cognitive instruction. Hillsdale : Erlbaum, p. 15-51.

PARIS S., LIPSON M. \& WIXSON K. (1983). « Becoming a strategic reader ». Contemporary Educational Psychology, vol. 8, n 3, p. 293-316.

PERKINS D., SIMMONS R. \& TISHMAN S. (1990). « Teaching cognitive and metacognitive strategies ». Journal of Structured Learning, vol. 10, n० 4, p. 285-303.

PINTRICH P. (1989). "The dynamic interplay of student motivation and cognition in the college classroom ". In C. Ames \& M. Maehr (dir.), Advances in motivation and achievement. Vol. 6: Motivation-enhancing environments. Greenwich : JAI Press, p. 117-160.

PINTRICH P. (1999). " The role of motivation in promoting and sustaining self-regulated learning ». International Journal of Educational Research, vol. 31, no 6, p. 459-470.

PINTRICH P. (2000). "The role of goal orientation in self-regulated learning ". In M. Boekaerts, P. Pintrich \& M. Zeidner (dir.), Handbook of self-regulation. San Diego : Academic Press, p. 451-502.

PINTRICH P. (2002). "The role of metacognitive knowledge in learning, teaching, and assessing ». Theory into Practice, vol. 41, n० 4, p. 219-225.

PINTRICH P. (2004). «A conceptual framework for assessing motivation and self-regulated learning in college students ». Educational Psychology Review, vol. 16, n० 4, p. 385407.

PINTRICH P. \& GARCIA T. (1991). « Student goal orientation and self-regulation in the college classroom ". In M. Maehr \& P. Pintrich (dir.), Advances in motivation and achievement, vol. 7. Greenwich : JAI Press, p. 371-402.

PINTRICH P. \& ZUSHO A. (2002). "The development of academic self-regulation: The role of cognitive and motivational factors ". In A. Wigfield \& J. Eccles (dir.), Development of achievement motivation. San Diego : Academic Press, p. 249-284.

PINTRICH P., MARX R. \& BOYLE R. (1993). «Beyond cold conceptual change: The role of motivational beliefs and classroom contextual factors in the process of conceptual change ». Review of Educational Research, vol. 63, no 2, p. 167-199. 
PINTRICH P., WOLTERS C. \& BAXTER G. (2000). « Assessing metacognition and selfregulated learning ". In G. Schraw \& J. Impara (dir.), Issues in the measurement of metacognition. Lincoln : Buros Institute, p. 43-97.

PINTRICH P., SMITH D., GARCIA T. \& MCKEACHIE W. (1993). «Reliability and predictive validity of the Motivated Strategies for Learning Questionnaire (MSLQ) ». Educational and Psychological Measurement, vol. 53, n 3, p. 801-813.

PRESSLEY M., BORKOWSKI J. \& SCHNEIDER W. (1987). "Cognitive strategies: Good strategy users coordinate metacognition and knowledge ". In R. Vasta \& G. Whitehurst (dir.), Annals of child development, vol. 55. Greenwich : JAI Press, p. 89-129.

PRESSLEY M., HARRIS K. \& MARKS M. (1992). "But good strategy instructors are constructivists! »Educational Psychology Review, vol. 4, no 1, p. 3-31.

REID M. \& BORKOWSKI J. (1987). "Causal attributions of hyperactive children: Implications for teaching strategies and self-control ». Journal of Educational Psychology, vol. 79, n०3, p. 296-307.

ROH S. (2012). Les apports de la métacognition face au défi de l'analyse de texte. Mémoire de maîtrise, sciences de l'éducation, Université de Genève, Suisse.

SCHRAW G. (1998). "Promoting general metacognitive awareness ». Instructional Science, vol. 26 , no $1-2$, p. 113-125.

SCHRAW G. \& DENNISON R. (1994). «Assessing metacognitive awareness ». Contemporary Educational Psychology, vol. 19, no 4, p. 460-475.

SCHRAW G., HORN C., THORNDIKE-CHRIST T. \& BRUNING R. (1995). "Academic goal orientations and student classroom achievement ". Contemporary Educational Psychology, vol. 20, no 3, p. 359-368.

SCHUNK D. (1982). "Verbal self-regulation as a facilitator of children's achievement and self-efficacy ». Human Learning, vol. 1, n², p. 265-277.

SCHUNK D. (1989). "Self-efficacy and cognitive skill learning ». In C. Ames \& R. Ames (dir.), Research on motivation in education. Vol. 3: Goals and cognitions. San Diego : Academic Press, p. 13-44.

SCHUNK D. \& ERTMER P. (2000). "Self-regulation and academic learning. Self-efficacy enhancing interventions ». In M. Boekaerts, P. Pintrich \& M. Zeidner (dir.), Handbook of self-regulation. San Diego : Academic Press, p. 631-649.

SCHUNK D. \& PAJARES F. (2005). «Competence perceptions and academic functioning ». In A. Elliot \& C. Dweck (dir.), Handbook of competence and motivation. New York : Guilford Press, p. 85-104.

SCHWARTZ B. \& PERFECT T. (2002). «Introduction: Toward an applied metacognition ». In T. Perfect \& B. Schwartz (dir.), Applied metacognition. Cambridge : University Press, p. 1-11.

SENKO C. \& MILES K. (2008). "Pursuing their own learning agenda: How mastery-oriented students jeopardize their class performance ». Contemporary Educational Psychology, vol. 33, no 4, p. 561-583.

SKINNER E. (1996). "A guide to constructs of control ». Journal of Personality and Social Psychology, vol. 71, n० 3, p. 549-570.

SKINNER E., ZIMMER-GEMBECK M. \& CONNELL J. (1998). «Individual differences and the development of perceived control ". Monographs of the Society for Research in Child Development, vol. 63, no 2-3, p. 1-220.

SON L. \& METCALFE J. (2000). "Metacognitive and control strategies in study-time allocation ". Journal of Experimental Psychology: Learning, Memory, and Cognition, vol. 26, no 1, p. 204-221.

SON L. \& SCHWARTZ B. (2002). "The relation between metacognitive monitoring and control ». In T. Perfect \& B. Schwartz (dir.), Applied metacognition. Cambridge : University Press, p. 15-38.

SPERLING R., HOWARD B., STALEY R. \& DUBOIS N. (2004). " Metacognition and selfregulated learning constructs ». Educational Research and Evaluation, vol. 10, n० 2, p. 117-139.

THOMAS G. (2003). "Conceptualisation, development and validation of an instrument for investigating the metacognitive orientation of science classroom learning environments: The Metacognitive Orientation Learning Environment Scale-Science (MOLES-S) ». Learning Environments Research, vol. 6, n² 2, p. 175-197. 
TOBIAS S. (1994). "Interest, prior knowledge, and learning ». Review of Educational Research, vol. 64, no 1, p. 37-54.

TOBIAS S. (1995). «Interest and metacognitive word knowledge ». Journal of Educational Psychology, vol. 87, n० 3, p. 399-405.

TOBIAS S. \& EVERSON H. (1997). "Studying the relationship between affective and metacognitive variables ». Anxiety, Stress, and Coping, vol. 10, no 1, p. 59-81.

TOBIAS S. \& EVERSON H. (2000). "Assessing metacognitive knowledge monitoring ". In G. Schraw \& J. Impara (dir.), Issues in the measurement of metacognition. Lincoln : Buros Institute, p. 147-222.

VandenBOS G. (2007). APA Dictionary of Psychology. Washington : American Psychological Association.

VEENMAN M., ELSHOUT J. \& MEIJER J. (1997). "The generality vs domain-specificity of metacognitive skills in novice learning across domains ». Learning and Instruction, vol. 7, no 2, p. 187-209.

VEENMAN M., KERSEBOOM L. \& IMTHORN C. (2000). «Test anxiety and metacognitive skillfulness: Availability versus production deficiencies ». Anxiety, Stress, and Coping, vol. 13, n० 4, p. 391-412.

WEINER B. (1986). An attributional theory of motivation and emotion. New York : SpringerVerlag.

WEINERT F. (1987). " Introduction and overview: Metacognition and motivation as determinants of effective learning and understanding ". In F. Weinert \& R. Kluwe (dir.), Metacognition, motivation, and understanding. Hillsdale : Erlbaum, p. 1-16.

WIGFIELD A. \& ECCLES J. (2002). «The development of competence beliefs, expectancies for success, and achievement values from childhood through adolescence ". In A. Wigfield \& J. Eccles (dir.), Development of achievement motivation. San Diego : Academic Press, p. 91-120.

WINNE P. \& HADWIN A. (1998). "Studying as self-regulated learning ». In D. Hacker, J. Dunlosky \& A. Graesser (dir.), Metacognition in educational theory and practice. Mahwah : Erlbaum, p. 277-304.

WOLTERS C. (2003). " Regulation of motivation: Evaluating an underemphasized aspect of self-regulated learning ». Educational Psychologist, vol. 38, n० 4, p. 189-205.

WOLTERS C. \& PINTRICH P. (1998). "Contextual differences in student motivation and self-regulated learning in mathematics, English, and social studies classrooms ». Instructional Science, vol. 26, no 1-2, p. 27-47.

WOLTERS C., PINTRICH P. \& KARABENICK S. (2005). « Measuring academic self-regulated learning ». In K. Moore \& L. Lippman (dir.), Conceptualizing and measuring indicators of positive development: What do children need to flourish? New York : Kluwer, p. 251279.

WOLTERS C., YU S. \& PINTRICH P. (1996). " The relation between goal orientation and student's motivational beliefs and self-regulated learning ". Learning and Individual Differences, vol. 8, n 3, p. 211-238.

ZAJONC R. (1980). "Feeling and thinking: Preferences need no inferences ». American Psychologist, vol. 35, no 2, p. 151-175.

ZIMMERMAN B. (2000). "Attaining self-regulation. A social cognitive perspective ». In M. Boekaerts, P. Pintrich \& M. Zeidner (dir.), Handbook of self-regulation. San Diego : Academic Press, p. 13-39.

ZIMMERMAN B. \& SCHUNK D. (2004). "Self-regulating intellectual processes and outcomes: A social cognitive perspective ». In D. Dai \& R. Sternberg (dir.), Motivation, emotion, and cognition. Integrative perspectives on intellectual functioning and development. Mahwah : Erlbaum, p. 323-349. 
